\title{
Spectral evolution of the recurrent nova RS Ophiuchi during the 2006 outburst and some mysterious phenomena ${ }^{\star}$
}

\author{
T. Iijima
}

\author{
Astronomical Observatory of Padova, Asiago Section, Osservatorio Astrofisico, 36012 Asiago (Vi), Italy \\ e-mail: takashi.iijima@oapd.inaf.it
}

Received 28 November 2008 / Accepted 26 June 2009

ABSTRACT

\begin{abstract}
Spectral evolution in the optical region of the recurrent nova RS Oph during the 2006 outburst was monitored at the Asiago Astrophysical Observatory. A short-lived flare-up of He I emission lines, which was clearly different from the helium flash of classical novae, was observed on March 7, that is 22 days after the outburst and 3.8 mag below maximum luminosity. The coronal emission line [Fe XIV] 5303 was first detected 35 days after the outburst. Its maximum intensity relative to $\mathrm{H} \beta$ was observed 80 days after the outburst, then the intensity declined rapidly. Another coronal line [Ar XI] 6919 showed a similar variation, while [Fe X] 6374 faded more slowly. When the former two coronal lines started to fade about 90 days after the outburst, the emission lines of [O I], [N II], [S II], and [O III] started to strengthen rapidly. Probably, the collisional shock front on the high velocity ejecta reached the outer boundary of the circumstellar envelope and a free expansion started at that time. The variations in intensity of the coronal lines [Fe XIV] and [Ar XI] coincided with that of the flux of super-soft X-rays, suggesting that also the latter originated in the collisional shock front. Many prominent emission lines were composed of a central narrow component, blue- and red-shifted wings, and a very broad component. Unidentified emission lines at 6105.5 and $6181.2 \AA$ are identified as O IV 4s $2 \mathrm{P}^{0}-4 \mathrm{p} 2 \mathrm{~S}, 6105.9$ and $6183.4 \AA$, respectively. On the other hand, new unidentified, probably coronal, emission lines are found at $7711 \pm 2 \AA$ and $7774 \pm 2 \AA$. A weak trace of the Raman emission band at $6830 \AA$ was detected when the emission lines of [Fe VII] were not seen. It seems that the lines of O IV at about $1033 \AA$ contributed, in the place of O VI $1032 \AA$, to the Raman scattering in a low ionization condition. Splittings in two parts of the narrow emission components of H I, He I, and the coronal lines were observed. The splittings were largest on April 8 , when the separations between the two peaks were $48 \mathrm{~km} \mathrm{~s}^{-1}$ for [Fe XIV] $5303,37 \mathrm{~km} \mathrm{~s}^{-1}$ for H $\beta$, and $33 \mathrm{~km} \mathrm{~s}^{-1}$ for $\mathrm{He}$ I 5876 . On the other hand, the narrow emission components of Fe II, [N II], [O III], O IV, and [Fe VII] did not show any splitting.
\end{abstract}

Key words. stars: individual: RS Ophiuchi - novae, cataclysmic variables - circumstellar matter

\section{Introduction}

The well known and best studied recurrent nova RS Oph underwent a new outburst in February 2006 with an interval of 21 years from the last one in 1985. The outburst was noticed by Narumi (2006) on February 12.83 UT, 2006 near maximum luminosity: $V=4.5 \mathrm{mag}$. We determine this as time zero, with a Julian date 2453779.3.

Numerous works studied the new outburst in multiple spectral regions, e.g., in X-rays (Bode et al. 2006; Ness et al. 2007; Vaytet et al. 2007), in optical (Bode et al. 2007), in infrared (Das et al. 2006; Chesneau et al. 2007; Evans et al. 2007a,b; Lane et al. 2007), and in radio wavelength (O'Brien et al. 2006). Some theoretical work on the explosion was carried out (e.g., Hachisu et al. 2006).

RS Oph is one of the main targets for the monitoring of the spectral evolution of novae and related objects in our observatory. The spectral evolution of the outburst in 1967 was reported by Barbon et al. (1969), and that of the outburst in 1985 by Rosino \& Iijima (1987). A review of the historical outbursts was performed by Rosino (1987).

Our first observation in the present outburst was made on February 18, 2006, namely 5.4 days after maximum luminosity, then the spectral evolution was monitored until the end of

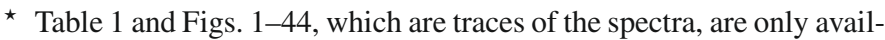
able in electronic form at http: //www . aanda.org
October 2006. In this paper, we report the spectral evolution of this object during the 2006 outburst with some interpretations (hereafter we will omit the term 2006 from the date).

\section{Observation}

High dispersion spectra with a resolution of $\lambda / \Delta \lambda \cong 15000$ were obtained with a Reosc Echelle spectrograph mounted on the $182 \mathrm{~cm}$ telescope at the Mount Ekar station of the Astronomical Observatory of Padova. These spectra are denoted as "Ech" in the log of the observations (Table 1). Medium dispersion spectra were obtained with a Boller \& Chivens grating spectrograph mounted on the $122 \mathrm{~cm}$ telescope at the Astrophysical Observatory of Asiago, affiliated to the University of Padova. The resolution was $\lambda / \Delta \lambda \cong 1000$ with a grating of 600 lines $\mathrm{mm}^{-1}$ which was used for most observations. Spectra obtained with this grating are denoted as "B\&C" in the log and several spectra obtained with another grating of 300 lines $\mathrm{mm}^{-1}$ are denoted as "B\&C3".

The spectra were reduced by using the standard tasks of the NOAO IRAF ${ }^{1}$ package at the Asiago Observatory. Spectrophotometric calibrations were completed with the spectra of standard stars obtained in the same nights. The standard stars HR4963 and HR5501 were used in the period from

\footnotetext{
1 IRAF is distributed by NOAO for Research in Astronomy, Inc. under cooperative agreement with the National Science Foundation.
} 
February to the end of April, then HR7596 and Kopff 27 were used in the later season. The effects of the interstellar extinction are corrected assuming $E(B-V)=0.71 \mathrm{mag}$ (Iijima 2008). The identifications of the emission lines were performed comparing the observed wavelengths with those given in the multiplet table of Moore (1959), the catalog of Meinel et al. (1968), and the list in the NIST atomic spectra database ${ }^{2}$. Intensities of selected emission lines were measured on the medium dispersion spectra (Sect. 4).

\section{Spectral evolution}

\subsection{February 18 and 19: days 5 and 6}

Our first spectra were obtained with the Echelle spectrograph on February 18.2 UT, that is 5.4 days after the outburst. The spectra covered the region from 5150 to $6860 \AA$ with some gaps owing to discontinuities between the orders. Unfortunately, we had to use a rather small CCD chip at that time. High dispersion spectra covering the region from 4200 to $5960 \AA$ were obtained the next day.

Figures 1-4 show the profiles of $\mathrm{H} \alpha$, He I 5876, [N II] 5755 , and some Fe II lines. The emission lines of $\mathrm{H} \alpha$ and $\mathrm{He}$ I 5876 consisted of a broad component and a weak narrow emission component accompanied by a P Cygni type narrow absorption. The absorption components were blue-shifted from the narrow emission components by $-45.7 \pm 0.7 \mathrm{~km} \mathrm{~s}^{-1}$ for $\mathrm{H} \alpha$ and $-27.6 \pm$ $0.7 \mathrm{~km} \mathrm{~s}^{-1}$ for He I 5876. The FWHMs of the broad components of $\mathrm{H} \alpha$ and $\mathrm{He}$ I 5876 were roughly $1800 \mathrm{~km} \mathrm{~s}^{-1}$. The emission line of [N II] 5755 had only a narrow component (Fig. 3), while both the narrow and broad components were prominent for the Fe II lines (Fig. 4).

Figures 5 and 6 show the profiles of $\mathrm{H} \beta$ and He I 5876 on February 19. The profile of He I 5876 was nearly the same as that on the day before (Fig. 2), excepting for the disappearance of the absorption component. The absorption component of $\mathrm{H} \beta$ was very weak (Fig. 5).

Figure 7 shows the spectral region $4620-4720 \AA$ A. The emission lines of Fe II were prominent and some lines of [Fe II] and [Fe III] were seen. The emission line of He II 4686 was very weak, with an equivalent width of roughly $0.04 \AA$, and a broad emission of He I 4713 was seen.

Figure 8 shows the spectral region $4930-5030 \AA$ A. The emission lines of [O III] 5007 and 4959 had only narrow components. The weak emission at $4993 \AA$ could be due to N II 4994.4 $\AA$. The narrow emission component of Fe II 42, 5018 was stronger than that of He I 5016. The emission of He I 5016, however, should have been dominant in the broad component, because the profile of the broad one was similar to that of He I 5876 (Fig. 6). The profiles of [N II] 5755 and the other Fe II lines are not presented, because they were nearly the same as those on the day before.

The peak of the broad emission component of He I 5876 was red-shifted with respect to the narrow component by $+243 \mathrm{~km} \mathrm{~s}^{-1}$ on February 18 (Fig. 2) and $+289 \mathrm{~km} \mathrm{~s}^{-1}$ on February 19 (Fig. 6). The line center of the broad component measured at half maximum of the height were also red-shifted by $+432 \mathrm{~km} \mathrm{~s}^{-1}$ and $+428 \mathrm{~km} \mathrm{~s}^{-1}$. On the other hand, the peak of the broad component of $\mathrm{H} \alpha$ on February 18 (Fig. 1) was redshifted by $+91 \mathrm{~km} \mathrm{~s}^{-1}$, while the line center at half maximum of the height was blue-shifted by $-30 \mathrm{~km} \mathrm{~s}^{-1}$. Those of $\mathrm{H} \beta$ on February 19 (Fig. 5) were $+202 \mathrm{~km} \mathrm{~s}^{-1}$, and $-28 \mathrm{~km} \mathrm{~s}^{-1}$, respectively. The observational errors in these velocities were about

\footnotetext{
${ }^{2}$ http://physics.nist.gov/cgi-bin/AtData/lines-form
}

$\pm 1 \mathrm{~km} \mathrm{~s}^{-1}$. These results suggest an asymmetric and complicated structure of the ejecta.

As seen in Figs. 2 and 6, Na I D1 and D2 had two pairs of narrow absorption components. The mean radial velocity of the deeper components was $+5.3 \pm 0.9 \mathrm{~km} \mathrm{~s}^{-1}$ in the frame of the local standard of rest. These components obviously belong to the interstellar matter. On the other hand, the mean radial velocity of the weaker components was $-52.8 \pm 0.9 \mathrm{~km} \mathrm{~s}^{-1}$, and there is no interstellar cloud with such a radial velocity in the direction of RS Oph (Kerr \& Westerhout 1965). The weaker absorption components were blue-shifted by $-33 \pm 1 \mathrm{~km} \mathrm{~s}^{-1}$ with respect to the system of this object (Iijima 2008). This velocity is possibly that of the stellar wind of a red giant. Thus, the weaker absorption components presumably belonged to the circumstellar envelope formed by mass outflow from the red giant in its quiet stage. A preliminary report on the circumstellar envelope was given by Iijima (2008), and a detailed argument will be made in a separate paper.

\subsection{March 6-14: days 21-29}

Our first medium dispersion spectra were obtained on March 6, 21.3 days after the outburst. The spectra covered the region from 3600 to $7350 \AA$ with a resolution of $\lambda / \Delta \lambda \cong 1000$. Traces of the blue and red spectra are shown in Figs. 9 and 10. The emission lines of He II were already prominent in the spectra, but those of highly ionized ions, e.g. [Fe VII] or coronal lines, were not seen yet. A weak trace of the Raman band at $6830 \AA$ (Schmid 1989) was seen (Fig. 10). Even in the medium dispersion spectra, it is possible to see that the emission line of [N II] 5755 had an intense broad component, while [O III] 4959 still had only a narrow component. This result suggests that the electron density of the ejecta was low enough to emit the [N II] line, but was too high for the [O III] lines.

Medium dispersion spectra obtained on March 7, 8, and 14 showed virtually the same spectral feature, excepting for the flare-up of He I lines on March 7 (Sect. 4.1).

\subsection{March 20: day 35}

High dispersion spectra were obtained on March 20, which covered the spectral region from 4200 to $5960 \AA$ again with some gaps.

Figure 11 shows the spectral region $4620-4720 \AA$. The emission line of He II 4686 was the strongest in this region. The emission lines of Fe II weakened, and N III 4641 was stronger than Fe II 37, 4629. Each of the emission lines of He II 4686 and N III 4641 was composed of a central narrow component, blue- and red-shifted emission wings, and a very broad emission component. Detailed arguments for the decomposition of the prominent emission lines are given in Sect. 5.2.

Figure 12 shows the profile of $\mathrm{H} \beta$. The Gaussian decomposition shows that there were also four emission components in $\mathrm{H} \beta$ (Sect. 5.2). However, the blue-shifted wing was merged in the central component.

Figure 13 shows the spectral region 4930-5030 A. The emission lines of [O III] 5007 and 4959 still showed only the narrow components. The intensity ratios of the narrow to broad components of He I 5016 and Fe II 42, 5018 greatly increased. In particular, the growth of the narrow component of He I 5016 was remarkable.

Figure 14 shows the spectral region 5280-5390 A. The coronal emission line [Fe XIV] 5303 was first detected in this 
spectrum. In contrast to the other forbidden lines, this line did not have the prominent narrow component in the first stage. The emission lines of Fe II were still prominent and some lines of [Fe II] were detected.

Figure 15 shows the spectral region 5690-5810 А. The broad component became dominant in the emission of [N II] 5755 , but the narrow component also was clearly visible. The emission line of [Fe VII] 5721 had only a narrow component. It is noteworthy that the profile of [Fe VII] 5721 was very different from that of [Fe XIV] 5303.

Figure 16 shows the profile of He I 5876. It seems that the profile of He I 5876 lay halfway between those of He II 4686 (Fig. 11) and $\mathrm{H} \beta$ (Fig. 12).

\subsection{April 4-25: days 50-71}

Figures 17 and 18 show the medium dispersion blue and red spectra obtained on April 4: day 50.3. Numerous coronal emission lines were seen in the spectra. The line of [Fe XIV] 5303 was much stronger than Fe II 49, 5317, and [Fe X] 6374 was one of the strongest emission lines in the red region after $\mathrm{H} \alpha$ (Fig. 18). The other coronal emission lines detected in these spectra were of [Fe XI] 3986, [Ni XII] 4231, [Ni XIII] 5116, [Ni $\mathrm{XV}]$ 6702, [Ar X] 5534, and [Ar XI] 6919. The growths in intensity of the broad emission components of [O III] 5007, 4959, and 4363 were remarkable, and the Raman band at $6830 \AA$ was strengthened. The emission line of [O I] 6300, which was hard to see in the previous spectra (e.g., Fig. 10), was clearly seen in this spectrum (Fig. 18).

A red spectrum obtained on April 24 covered the spectral region up to $7925 \AA$ (Fig. 19). The coronal emission line [Fe XI] $7892 \AA$ was prominent in the reddest part of the spectrum. The identifications of two emission lines at 7711 and $7774 \AA$, and a broad emission complex at $7322 \AA$ are discussed in Sect. 4.3.

High dispersion spectra were obtained on April 8: day 54.3. Figure 20 shows the spectral region 4620-4720 A. Notable changes from the spectrum on March 20 (Fig. 11) were the fading of the emission wings of He II 4686 and N III 4641.

Figure 21 shows the profile of $\mathrm{H} \beta$. Also the emission wings of $\mathrm{H} \beta$ faded. One unexpected finding is that the central "narrow" component of $\mathrm{H} \beta$ was obviously broader than those of He II 4686, N III 4641 (Fig. 20), or He I 4922. This phenomenon is discussed in Sect. 5.3.

Figure 22 shows the spectral region $4930-5030 \AA$. The emission lines of [O III] 5007 and 4959 had narrow components and very broad emission components, but no blue- or red-shifted wing. The electron density in the emitting regions of the wings, which were the slowly expanding parts of the ejecta, were probably still too high for the [O III] lines.

Figure 23 shows the spectral region 5280-5390 $\AA$. It is easy to see that the narrow component of [Fe XIV] 5303 was split in two parts. The separation between the two peaks was $48 \pm 1 \mathrm{~km} \mathrm{~s}^{-1}$. On the other hand, the narrow components of the Fe II lines did not show any splitting or broadening. A detailed argument for the splittings of the narrow components is given in Sect. 5.3.

Figure 24 shows the spectral region 5690-5810 A. The profile of [N II] 5755 was very different from those of the [O III] lines (Fig. 22), and rather resembled that of $\mathrm{H} \beta$ (Fig. 21). The profile of [Fe VII] 5721 was similar to those of the [O III] lines. A weak trace of a coronal line [Ca XV] 5694 was seen.

Figure 25 shows the spectral region 5840-5960 А. The profile of He I 5876 again lay between those of He II 4686 (Fig. 20) and $\mathrm{H} \beta$ (Fig. 21). Weak emission components emerged on the redder sides of the weaker absorption lines of Na I D1 and D2. This phenomenon supports the hypothesis of the circumstellar origin of these absorption lines.

\subsection{May 7-June 4: days 83-111}

High dispersion spectra were obtained on May 17: day 93.2. Figure 26 shows the profile of He II 4686. The emission wings, in particular the red one, faded. The peak of the blue-ward wing was shifted by $-140 \mathrm{~km} \mathrm{~s}^{-1}$ with respect to the central component. This spectrum did not cover the spectral region of N III 4641.

Figure 27 shows the profile of $\mathrm{H} \beta$. The fading of the wings was great in $\mathrm{H} \beta$ than in He II 4686 (Fig. 26) or in He I 5876 which is shown later. The splitting of the narrow component decreased (see Sect. 5.3).

Figure 28 shows the spectral region 4930-5030 A. The broad components of [O III] lines strengthened, while their narrow components weakened. The emission feature at about $5004 \AA$ was identified as a blue-ward wing of [O III] 5007 shifted by $-133 \mathrm{~km} \mathrm{~s}^{-1}$. The emission line of He I 5016 was stronger than that of Fe II 5018.

Figure 29 shows the spectral region 5280-5390 A. The emission wings of [Fe XIV] 5303 as well those of Fe II lines nearly disappeared. The narrow emission component of [Fe XIV] 5303 was obviously broader than that of Fe II 49, 5317, but the clear splitting such as on April 8 (Fig. 23) was not seen.

Figure 30 shows the spectral region 5690-5810 A. The narrow component of [N II] 5755 weakened, and that of [Fe VII] 5721 was nearly embedded in the noise. The blue-ward wing of [N II] 5755 was shifted by $-85 \mathrm{~km} \mathrm{~s}^{-1}$.

Figure 31 shows the spectral region 5840-5955 A. The emission wings of He I 5876 also faded. This profile did not lie halfway between those of $\mathrm{He}$ II 4686 (Fig. 26) and $\mathrm{H} \beta$ (Fig. 27), but resembled that of He II 4686. The displacements of the blue and red wings of He I 5876 with respect to the narrow component were $-158 \mathrm{~km} \mathrm{~s}^{-1}$ and $+153 \mathrm{~km} \mathrm{~s}^{-1}$. The emission components of the circumstellar Na I D1 and D2 lines were prominent.

Figure 32 shows the spectral region 6000-6120 $\AA$. The emission line at $6106 \AA$ was prominent. This line is identified as O IV $6105.9 \AA$ (see Sect. 4.3). The emission line [Fe VII] 6086 showed a central narrow component, a blue-ward wing, and a very broad component. The blue-ward wing, shifted by $-146 \mathrm{~km} \mathrm{~s}^{-1}$, was stronger than the central narrow component.

Figure 33 shows the spectral region $6340-6470 \AA$. The emission line $[\mathrm{Fe} \mathrm{X}] 6374$ was prominent in this region. In contrast to [Fe XIV] 5303, blue and red wings, shifted by $-127 \mathrm{~km} \mathrm{~s}^{-1}$ and $+63 \mathrm{~km} \mathrm{~s}^{-1}$, were clearly seen. The blue-shifted wing was blended by Si II 6371, but the contribution of the latter was expected to have been very small, because the line of Si II 6347 was so weak. The other emission lines were of Fe II.

Medium dispersion spectra obtained on May 7 showed nearly the same spectral features as those on April 4 (Figs. 17, 18). Large changes were noticed on May 25 and 26, 102 days after the outburst. Figure 34 shows a trace of the blue spectrum obtained on May 26, where the strengthening of the emission lines of [N II], [Ne III], [O III], and [Fe VII] was notable; meanwhile the coronal line [Fe XIV] 5303 faded. In the red spectrum obtained on May 25 (Fig. 35) the coronal line [Fe X] 6374 was still intense, but the other coronal line [Ar XI] 6919 as well the emission line at $7711 \AA$ nearly disappeared. Also, during 
the 1985 outburst, most of the coronal lines nearly disappeared about 100 days after the outburst (Wallerstein \& Garnavich 1986; Rosino \& Iijima 1987). The strengthening of the emission lines of [Fe VII] 6086, [O I] 6300, and [Fe II] 7155 was notable in the red region. The broad emission complex at $7322 \AA$ grew much in intensity, and three peaks were observed of wavelengths, 7328, and $7340 \AA$ with errors of $\pm 2 \AA$ (see Sect. 4.3).

Medium dispersion spectra obtained on June 3 and 4 showed nearly the same features as those on May 25 and 26.

\subsection{June 10 and later: days 117-}

High dispersion spectra were obtained on June 10, in which the coronal line [Fe XIV] 5303 nearly disappeared, while the other spectral features resembled those on May 17. Several high dispersion spectra were obtained in August 30, 31, September 1, and 5. Here, we present that of August 31: day 200.1.

Figure 36 shows the spectral region $4620-4720 \AA$. The very broad component and the emission wings of He II 4686 disappeared. Since the signal level was low, it was difficult to decide whether the narrow emission components of He II 4686 and N III 4640 were split in two parts or those features were due to noise. The weak emission lines of N III 4634, Fe II 4629, and 4667 were detected.

Figure 37 shows the profile of $\mathrm{H} \beta$. The wide spread emission tails were barely visible. A secondary emission component, blue-shifted by $-83 \mathrm{~km} \mathrm{~s}^{-1}$ with respect to the main component, was seen. The character of this component was different from those of the blue-shifted emission wings observed in the earlier stages. For example, it was much narrower than the emission wings. In addition to the difference in profile, the emission wings in the earlier stages were more clearly seen in the lines of He II and He I, but a similar secondary component was not seen in He II 4686 (Fig. 36) or in He I 5876 as seen later. Similar secondary emission components were seen in the H I lines on August 30, September 1, and September 5, but was not seen on August 7.

Figure 38 shows the spectral region 4930-5030 ̊. The clear narrow emission component of [O III] 5007 was not seen, and two rather narrow components with similar widths, which are indicated by vertical lines, were superimposed on the very broad emission component. The emission line of [O III] 4959 showed a similar profile and the mean separation between the two rather narrow components was about $150 \mathrm{~km} \mathrm{~s}^{-1}$. The narrow emission component of He I 5016 weakened.

Figure 39 shows the spectral region 5280-5390 A. The strongest emission line in this region was Fe II 49, 5316. The coronal line [Fe XIV] 5303 seems to have disappeared. A weak emission feature at $5304 \AA$ may have been due to O IV 11 , 5305.3 , but this identification was not confirmed, because the other line of O IV of the same multiplet at 5362.4 $\AA$ (Moore 1959) was blended by Fe II 48, 5362.9.

Figure 40 shows the spectral region 5690-5805 $\AA$. As for the [O III] lines, [N II] 5755 had a very broad emission component and two rather narrow emission components, indicated by vertical lines, with a separation of about $150 \mathrm{~km} \mathrm{~s}^{-1}$.

Figure 41 shows the spectral region 5840-5960 A. The very broad emission component and the emission wings of He I 5876 disappeared. A weak hump was seen at the redder side of the narrow emission component, which suggests that there was a secondary narrow component red-shifted by about $37 \mathrm{~km} \mathrm{~s}^{-1}$.

The emission components of the circumstellar Na I D1 and D2 lines disappeared. The equivalent widths of these absorption lines were roughly the same as those in the spectra in the early stage (Figs. 2, 6). This result suggests that the circumstellar envelope along the line of sight was not disturbed by the outburst. Only a part of the circumstellar envelope seems to have been disturbed by the high velocity gas outflow on the outburst.

Medium dispersion spectra were obtained on September 9 and 12. The blue spectrum on September 9 is shown in Fig. 42. The emission line of [O III] 4363 weakened with respect to those of [O III] 5007 and 4959, which means that the electron density in the ejecta decreased (Osterbrock 1989). The emission lines of [Fe II] developed and those of He II weakened with respect to $\mathrm{H}$ I lines.

Figure 43 shows a trace of the red spectrum on September 12. The coronal line [Fe X] 6374 disappeared. The weak emission feature at $6370 \AA$ was probably due to Si II 6371.4 and Fe II 6369.4. Weak traces of [Fe VII] 5721 and 6086 were seen, but it was hard to see whether there was the Raman band at $6830 \AA$ or not. The band heads of TiO molecules of the underlying M Type giant were seen at 5847, $6159 \AA$ etc. The emission complex at $7322 \AA$ was prominent.

Our last observation in 2006 was carried out on October 29: 259 days after the outburst, when a medium dispersion blue spectrum was obtained. Figure 44 shows its trace. The emission lines of He II, [O III], [Ne III], and [N II] largely faded. On the other hand, some emission lines had their highest intensities relative to $\mathrm{H} \beta$ in the last spectrum, those were $\mathrm{He}$ I 4388, 4471; Fe II 4584, 5232, 5317; [Fe II] 4415; and He I+Fe II 4922 (Table 2). These results reflected the low surface temperature of the ionizing source. The $\mathrm{TiO}$ absorption bands were seen at 5167 and $5448 \AA$.

\section{Variations of the intensities of emission lines}

Table 2 gives intensities of selected emission lines relative to $\mathrm{H} \beta=100$ measured in the medium dispersion spectra. Absolute intensities of $\mathrm{H} \beta$ were measured in the spectra obtained in photometric nights, and are given in the table in a unit of $10^{-10} \mathrm{erg} \mathrm{cm}^{-2} \mathrm{~s}^{-1}$. These values are corrected for the interstellar extinction assuming $E(B-V)=0.71 \mathrm{mag}$ (Iijima 2008). The observational errors in the relative intensities are roughly $10 \%$, but larger errors are expected for intensities of less than 2.0. The values of lower accuracies, which were mainly due to blending with other lines, are denoted by a colon. The errors in the absolute intensities of $\mathrm{H} \beta$ are also about $10 \%$. Three dots in the table mean that the line was not detected, that is, its intensity was less than about $0.1 \%$ of $\mathrm{H} \beta$. The blanks in the table mean that the corresponding spectral region was not observed, or was not calibrated because of some technical problems. In the table [K V],[S III] 6315 is a blend of [S III] 3F, 6310 and [K V] 2F, 6317. It seems that the line of [S III] became dominant in May and later. Since the spectral evolution was slow in September, the intensities measured in the spectra obtained on September 9 and 12 are combined and are given as those of September 9 (09/9). Figures $45-47$ show the variations of the intensities of selected emission lines.

\subsection{Flare-up of He I lines}

The first remarkable phenomenon in the variation of the emission lines in intensity was the sudden strengthening of He I emission lines on March 7 (Figs. 45, 47). The variations of the related emission lines are shown in detail in Fig. 48. As seen in Fig. 48 and Table 2, when the emission lines of He I 
Table 2. Intensities relative to $\mathrm{H} \beta=100$ of selected emission lines and absolute intensities of $\mathrm{H} \beta$ of RS Oph.

\begin{tabular}{|c|c|c|c|c|c|c|c|c|}
\hline 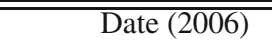 & $\overline{006 / 3}$ & $\overline{007 / 3}$ & $\overline{08 / 3}$ & $\overline{14 / 3}$ & $\overline{004 / 4}$ & $\overline{12 / 4}$ & $\overline{13 / 4}$ & $\overline{14 / 4}$ \\
\hline JD2450000+ & 3800.6 & 3801.7 & 3802.7 & 3808.7 & 3829.7 & 3837.6 & 3838.6 & 3839.6 \\
\hline $\mathrm{I}(\mathrm{H} \beta)(\mathrm{E}-10)$ & 38.0 & 32.9 & & 26.0 & 8.6 & 6.0 & & 4.9 \\
\hline [Ne V] 3426.6 & & & & & & & & \\
\hline O III 3444.1 & & & & 5.0 & & 16.67 & & \\
\hline [Fe VII $] 3760$ & $\ldots$ & $\ldots$ & $\ldots$ & 4.5 & 8.6 & 8.8 & 13.2 & 14.0 \\
\hline$[\mathrm{Ne}$ III $] 3868$ & $\cdots$ & 1.4 & 1.6 & 2.0 & 22.8 & 17.5 & 27.3 & 23.5 \\
\hline H I,He I 3889 & 27.6 & 24.6 & 23.8 & 19.7 & 27.7 & 25.0 & 33.5 & 26.1 \\
\hline H I,[Ne III] 3970 & 19.8 & 18.5 & 18.7 & 17.8 & 22.6 & 21.0 & 27.4 & 24.2 \\
\hline [Fe XI] 3986 & $\cdots$ & $\cdots$ & $\cdots$ & $\cdots$ & 3.1 & 3.0 & 4.0 & 2.2 \\
\hline He I,He II 4026 & 5.1 & 4.2 & 3.9 & 5.2 & 5.0 & 4.7 & 5.7 & 4.8 \\
\hline [S II] 4070 & 3.3 & 2.6 & 3.2: & 2.9 & 2.7 & 2.8 & 2.9 & 2.6 \\
\hline $\mathrm{H} \delta$ & 43.1 & 37.5 & 41.4 & 39.7 & 40.1 & 38.6 & 44.0 & 40.2 \\
\hline Fe II,[Fe II] 4180 & 3.1 & 2.8 & 1.9: & 2.3 & 3.2 & 3.5 & 4.2 & 4.0 \\
\hline Fe II,[NiXII]4233 & 3.5 & 2.7 & 3.3 & 2.7 & 5.4 & 5.9 & 6.5 & 6.9 \\
\hline [Fe II] 4244 & $\cdots$ & $\ldots$ & $\cdots$ & $\cdots$ & 1.2 & 1.2 & 1.6 & 0.7: \\
\hline $\mathrm{H} \gamma$ & 53.8 & 48.5 & 45.1 & 48.5 & 49.0 & 54.8 & 52.0 & 53.5 \\
\hline [O III] 4363 & & & & 3.2: & 28.7 & 33.2 & 34.5 & 34.4 \\
\hline He I 4388 & 2.9 & 4.7 & 4.7 & 3.8 & 2.5 & 2.6 & 3.1 & 1.7 \\
\hline [Fe II] 4415 & 1.4 & 1.5 & 1.1 & 1.5 & 3.0 & 3.5 & 3.4 & 3.0 \\
\hline He I 4472 & 7.7 & 8.3 & 8.1 & 7.2 & 7.1 & 7.3 & 7.3 & 6.9 \\
\hline Fe II 4584 & 3.2 & 2.8 & 2.6 & 2.6 & 3.8 & 4.2 & 4.1 & 3.6 \\
\hline N III 4634,4641 & 22.8 & 28.9 & 26.9 & 28.6 & 27.6 & 28.6 & 27.4 & 26.3 \\
\hline He II 4685.7 & 27.0 & 28.0 & 33.0 & 36.8 & 51.9 & 57.7 & 59.0 & 60.2 \\
\hline He I 4713,[ArIV] & 2.3 & 3.3 & 2.3 & 2.3 & 2.4 & 2.6 & 3.2 & 4.1: \\
\hline He I, Fe II 4922 & 7.2 & 9.2 & 8.6 & 7.1 & 6.2 & 6.6 & 6.5 & 6.7 \\
\hline [O III] 4959 & 0.4 & 0.3 & 0.3 & 0.3: & 5.0 & 6.0 & 6.1 & 6.0 \\
\hline [O III] 5007 & 1.4: & 2.2: & 2.1: & 2.2: & 13.2 & 12.9 & 12.5 & 11.6: \\
\hline He I, Fe II 5016 & 16.7 & 16.3 & 15.7 & 13.5 & 13.6 & 16.1 & 15.0 & 17.3 \\
\hline Si II 5041 & 4.7: & 3.8 & 4.4 & 4.8 & 8.9 & 7.8 & 8.0 & 8.4 \\
\hline Si II 5056 & 7.6: & 6.4 & 7.1 & 6.1 & 5.6 & 5.3 & 4.6 & 4.4 \\
\hline [Ni XIII] 5116 & $\cdots$ & $\cdots$ & $\cdots$ & $\cdots$ & 0.9 & 1.1 & 1.3 & 1.2 \\
\hline [Fe II],[Fe VII] 5158 & $\cdots$ & $\cdots$ & $\cdots$ & $\cdots$ & $\cdots$ & 1.3 & 1.3 & 1.2: \\
\hline Fe II 5169 & 6.2 & 5.5 & 6.0 & 5.1 & 4.4 & 4.6 & 4.2 & 6.9 \\
\hline Fe II 5235 & 1.5 & 1.4 & 1.4 & 1.3 & 2.3 & 2.6 & 2.5 & 2.4 \\
\hline Fe II 5276 & 2.4 & 1.9 & 2.2 & 1.9 & 2.1 & 2.2 & 2.0 & 4.0 \\
\hline [Fe XIV] 5303 & $\cdots$ & $\cdots$ & $\cdots$ & $\cdots$ & 9.9 & 12.7 & 12.3 & 14.7 \\
\hline Fe II 5317 & 4.2 & 3.6 & 3.5 & 4.1 & 4.0 & 4.2 & 3.8 & 3.9 \\
\hline He II 5412 & 2.8 & 3.2 & 3.5 & 3.9 & 4.3 & 4.2 & 4.2 & 5.6 \\
\hline [Fe II] 5496 & 0.8 & 0.6 & 0.5 & 0.6 & 0.7 & 0.8 & 0.7 & 0.7 \\
\hline N II,[Ar X] 5535 & 1.7 & 1.3 & 1.2 & 2.0 & 9.9 & 10.5 & 10.2 & 11.4 \\
\hline N II,[Fe VI] 5679 & 2.8 & 2.9 & 2.3 & 2.3 & 1.4 & 0.8 & 0.7 & 0.7 \\
\hline [Fe VII] 5721 & $\cdots$ & $\ldots$ & $\ldots$ & $\cdots$ & 1.2 & 1.4 & 1.3 & 1.5 \\
\hline [N II] 5755 & 2.5 & 2.0 & 2.0 & 3.2 & 6.7 & 7.1 & 6.5 & 6.6 \\
\hline He I 5876 & 38.6 & 50.3 & 44.3 & 38.3 & 31.8 & 31.8 & 30.7 & 32.7 \\
\hline [Fe VII] 6086 & $\cdots$ & $\ldots$ & $\cdots$ & 0.3 & 1.9 & 2.1 & 2.1 & 2.3 \\
\hline O IV 6106 & 1.3 & 0.3 & 1.0 & 1.5 & 1.6 & 1.7 & 1.6 & 2.0 \\
\hline O IV 6182 & 0.2 & $\cdots$ & $\cdots$ & 0.3 & 0.4 & 0.5 & 0.5 & 0.6 \\
\hline Fe II 6248 & 0.6 & 0.5 & 1.2 & 0.8 & 0.8 & 1.0 & 0.9 & 1.0 \\
\hline [O I] 6300 & 0.1 & 0.1 & $\cdots$ & 0.2 & 0.9 & 1.1 & 1.2 & 1.5 \\
\hline [K V],[S III]6315 & 0.6 & 0.3 & 0.5 & 0.6 & 1.2 & 1.2 & 1.3 & 1.2 \\
\hline Si II 6347 & 2.2 & 1.9 & 2.0 & 2.1 & 1.8 & 2.1 & 2.0 & 2.2 \\
\hline Si II,[Fe X] 6374 & 4.0 & 2.8 & 3.3 & 6.0 & 32.9 & 39.3 & 39.7 & 42.9 \\
\hline $\mathrm{H} \alpha$ & 593 & 636 & 626 & 609 & 653 & 636 & 640 & 671 \\
\hline He I 6678 & 11.6 & 20.9 & 16.8 & 11.7 & 10.0 & 11.2 & 11.4 & 12.1 \\
\hline [Ni XV] 6702 & $\ldots$ & $\ldots$ & $\ldots$ & $\ldots$ & 0.4 & 0.5 & 0.6 & 0.6 \\
\hline Raman 6830 & 0.6 & $\ldots$ & 1.1 & 1.5 & 4.3 & 4.8 & 4.6 & 5.3 \\
\hline [Ar XI] 6919 & $\cdots$ & $\ldots$ & $\cdots$ & $\cdots$ & 2.6 & 3.0 & 2.7 & 3.0 \\
\hline He I 7065 & 21.8 & 33.6 & 31.0 & 26.0 & 23.7 & 24.7 & 24.7 & 28.0 \\
\hline Raman 7088 & $\cdots$ & $\cdots$ & $\cdots$ & $\cdots$ & 0.2 & 0.4 & 0.3 & 0.3: \\
\hline [Fe II] 7155 & $\cdots$ & $\cdots$ & $\cdots$ & $\cdots$ & 0.3 & 0.4 & 0.4 & 0.5 \\
\hline He I 7281 & 2.3 & 5.0 & 3.8 & 2.2 & 2.2 & 2.7 & 2.9 & 2.6 \\
\hline uid. 7711 & & & & & 2.5 & 2.2 & 2.3 & 3.5 \\
\hline uid. 7774 & & & & & & 2.1 & 2.3 & 2.3 \\
\hline$[\mathrm{Fe} X \mathrm{XI}] 7890$ & & & & & & 19.8 & 20.3 & 22.4 \\
\hline
\end{tabular}


Table 2. continued.

\begin{tabular}{|c|c|c|c|c|c|c|c|c|c|}
\hline$\overline{\text { Date (2006) }}$ & $23 / 4$ & $24 / 4$ & $\overline{25 / 4}$ & $\overline{07 / 5}$ & $\overline{25 / 5}$ & $\overline{04 / 6}$ & $21 / 8$ & $\overline{09 / 9}$ & $29 / 10$ \\
\hline JD2450000+ & 3848.6 & 3849.6 & 3850.6 & 3862.6 & 3881.5 & 3890.5 & 3969.4 & 3988.4 & 4038.2 \\
\hline $\mathrm{I}(\mathrm{H} \beta)(\mathrm{E}-10)$ & 4.3 & & 3.1 & 2.6 & 1.2 & & 0.34 & 0.29 & 0.16 \\
\hline$[\mathrm{Ne} \mathrm{V}] 3426.6$ & 13.5 & & & & & & 5.2 & & \\
\hline O III 3444.1 & 23.2 & & & & & & 9.4 & & \\
\hline [Fe VII] 3760 & 14.1 & 9.9: & 12.8 & 11.4 & 11.3 & & 6.3 & 4.4 & \\
\hline [Ne III] 3868 & 23.7 & 20.3: & 22.6 & 35.6 & 51.3 & & 27.3 & 21.5 & 15.6 \\
\hline H I,He I 3889 & 26.9 & 27.5 & 26.5 & 31.7 & 33.4 & 33.9 & 18.3 & 17.6 & 16.5 \\
\hline H I,[Ne III] 3970 & 23.4 & 24.3 & 24.6 & 27.6 & 31.1 & 34.3 & 26.1 & 22.6 & 18.1 \\
\hline [Fe XI] 3986 & 2.7 & 4.5 & 4.0 & 4.1 & 2.6 & 3.1 & $\cdots$ & $\ldots$ & $\cdots$ \\
\hline He I,He II 4026 & 4.3 & 5.0 & 5.2 & 5.2 & 3.8 & 3.8 & 4.8 & 4.1 & 5.0 \\
\hline [S II] 4070 & 1.7 & 3.2 & 3.5 & 4.2 & 5.5 & 7.0 & 9.8 & 7.9 & 9.7 \\
\hline $\mathrm{H} \delta$ & 40.4 & 38.6 & 40.9 & 40.9 & 37.1 & 34.1 & 35.9 & 29.9 & 23.1 \\
\hline Fe II,[Fe II] 4180 & 4.4 & 2.9 & 2.8 & 4.5 & 3.6 & 4.0 & 3.2 & 2.9 & 3.3 \\
\hline Fe II,[NiXII]4233 & 7.2 & 6.3 & 6.6 & 6.8 & 4.0 & 3.1 & 3.7: & 2.3 & 3.7 \\
\hline [Fe II] 4244 & 1.3 & 1.9 & 2.3 & 3.2 & 6.3 & 7.6 & 5.2: & 4.7 & 5.4 \\
\hline $\mathrm{H} \gamma$ & 50.0 & 49.3 & 50.7 & 50.8 & 54.3 & 53.0 & 44.5 & 39.6 & 32.5 \\
\hline [O III] 4363 & 40.0 & 39.8 & 40.9 & 50.2 & 75.5 & 72.8 & 19.6 & 11.4 & 1.8 \\
\hline He I 4388 & 2.0 & 2.8 & 3.0 & 3.6 & 2.4 & 1.8: & 2.8 & 3.6 & 5.7 \\
\hline [Fe II] 4415 & 3.3 & 3.5 & 3.8 & 4.7 & 6.7 & 6.9 & 7.0 & 6.8 & 10.2 \\
\hline He I 4472 & 7.0 & 7.1 & 6.7 & 7.8 & 8.4 & 7.0 & 7.2 & 8.2 & 8.9 \\
\hline Fe II 4584 & 3.8 & 4.1 & 3.7 & 4.7 & 3.5 & 3.6 & 2.4 & 2.6 & 7.2 \\
\hline N III 4634,4641 & 25.9 & 25.4 & 25.9 & 25.7 & 18.3 & 15.1 & 26.0 & 20.6 & 13.1 \\
\hline He II 4685.7 & 61.0 & 64.5 & 67.3 & 71.2 & 62.1 & 60.2 & 52.8 & 46.1 & 17.2 \\
\hline He I 4713,[ArIV] & 4.2 & 3.1 & 3.2 & 3.3 & 2.9 & 2.7 & 3.0 & 3.9 & 4.1 \\
\hline He I, Fe II 4922 & 7.1 & 6.7 & 7.1 & 7.2 & 6.2 & 5.3 & 9.5 & 9.0 & 11.3 \\
\hline [O III] 4959 & 7.3 & 8.4 & 8.5 & 13.5 & 26.2 & 34.5 & 33.9 & 27.0 & 18.8 \\
\hline [O III] 5007 & 13.7 & 16.5 & 16.7 & 26.7 & 54.6 & 71.1 & 81.6 & 70.6 & 55.9 \\
\hline He I, Fe II 5016 & 18.1 & 16.6 & 16.7 & 20.7 & 27.5 & 29.8 & 21.9: & 22.1 & 20.7 \\
\hline Si II 5041 & 7.5 & 6.8 & 6.2 & 6.8 & 5.3 & 4.6 & 5.8 & 5.3 & 3.7 \\
\hline Si II 5056 & 4.2 & 4.3 & 4.3 & 3.9 & 4.1 & 4.1 & 5.3 & 6.5 & 5.9 \\
\hline [Ni XIII] 5116 & 1.3 & 1.5 & 1.7 & 1.8 & 1.1 & 0.7 & 1.5 & 0.9 & 0.3: \\
\hline$[\mathrm{Fe}$ II],Fe VII] 5158 & 0.9: & 1.8 & 1.7 & 3.1 & 6.2 & 8.3 & 10.5 & 6.6 & 8.6 \\
\hline Fe II 5169 & 7.1 & 5.3 & 5.2 & 5.6 & 4.8 & 4.8 & 1.2: & 1.6 & 4.3 \\
\hline Fe II 5235 & 2.7 & 2.8 & 2.7 & 2.7 & 2.6 & 2.8 & 3.9 & 4.0 & 6.8 \\
\hline Fe II 5276 & 3.2 & 2.5 & 2.9 & 3.3 & 4.9 & 6.4 & 6.1 & 3.9 & 4.5 \\
\hline [Fe XIV] 5303 & 16.5 & 15.3 & 15.8 & 17.4 & 3.5 & 0.6 & 0.6 : & 0.5 & 0.6 \\
\hline Fe II 5317 & 3.9 & 4.0 & 4.4 & 4.4 & 3.9 & 3.8 & 4.3 & 3.7 & 5.6 \\
\hline He II 5412 & 6.0 & 4.0 & 4.7 & 5.4 & 7.1 & 6.3 & 6.5 & 4.1 & 1.9 \\
\hline [Fe II] 5496 & 0.7 & 0.7 & 0.6 & 0.8 & 1.0 & 1.2 & 1.3 & 1.0 & 1.1 \\
\hline N II, $[$ Ar X] 5535 & 12.7 & 12.1 & 12.2 & 13.1 & 5.9 & 3.2 & 3.6 & 1.3 & 3.0 \\
\hline N II,[Fe VI] 5679 & 0.7 & 0.8 & 0.7 & 0.8 & 1.3 & 1.3 & 2.7 & 1.4 & 2.3 \\
\hline [Fe VII] 5721 & 1.8 & 1.9 & 2.1 & 3.3 & 5.6 & 5.5 & 3.5 & 1.8 & 1.2 \\
\hline [N II] 5755 & 7.3 & 8.3 & 8.3 & 12.9 & 35.7 & 43.3 & 46.4 & 36.8 & 32.3 \\
\hline He I 5876 & 32.2 & 31.8 & 30.7 & 33.6 & 32.0 & 29.6 & 31.3 & 33.2 & 38.2 \\
\hline [Fe VII] 6086 & 2.5 & 3.3 & 3.2 & 4.6 & 6.6 & 4.4 & 0.8 & 0.7 & \\
\hline O IV 6106 & 2.1 & 1.9 & 2.0 & 2.6 & 2.3 & 1.9 & 0.5 & 0.9 & \\
\hline O IV 6182 & 0.5 & 0.5 & 0.5 & 0.6 & 0.6 & 0.5 & $\cdots$ & $\cdots$ & \\
\hline Fe II 6248 & 1.1 & 1.0 & 1.0 & 1.1 & 0.9 & 0.5 & 1.0 & 1.3 & \\
\hline [O I] 6300 & 1.8 & 1.7 & 1.6 & 3.1 & 7.6 & 9.9 & 13.8 & 9.5 & \\
\hline [K V],[S III]6315 & 1.3 & 1.1 & 1.0 & 1.2 & 1.9 & 1.4 & 2.0: & 1.8 & \\
\hline Si II 6347 & 2.0 & 2.1 & 2.0 & 2.2 & 1.3 & 1.1 & 1.7 & 1.9 & \\
\hline Si II,[Fe X]6374 & 47.4 & 46.3 & 47.8 & 55.4 & 47.5 & 33.4 & 6.9 & 3.3 & \\
\hline $\mathrm{H} \alpha$ & 696 & 611 & 647 & 651 & 558 & 530 & 616 & 510 & \\
\hline He I 6678 & 13.1 & 12.2 & 12.2 & 14.2 & 13.0 & 11.8 & 21.7 & 18.3 & \\
\hline [Ni XV] 6702 & 0.6 & 0.7 & 0.7 & 0.7 & $\ldots$ & $\cdots$ & $\ldots$ & $\cdots$ & \\
\hline Raman 6830 & 5.9 & 5.4 & 5.7 & 6.3 & 6.1 & 4.8 & 3.1 & $\cdots$ & \\
\hline [Ar XI] 6919 & 3.5 & 3.4 & 3.5 & 3.2 & 0.6 & $\cdots$ & $\cdots$ & $\cdots$ & \\
\hline He I 7065 & 27.8 & 26.5 & 27.6 & 30.6 & 26.3 & 21.9 & 23.6 & 26.6 & \\
\hline Raman 7088 & $0.4:$ & 0.7 & 0.6 & 0.4 & $\cdots$ & $\cdots$ & $\cdots$ & $\cdots$ & \\
\hline [Fe II] 7155 & 0.6 & 0.6 & 0.6 & 1.1 & 2.8 & 3.1 & 5.3 & 4.1 & \\
\hline He I 7281 & 3.0 & 3.2 & 3.3 & 3.6 & 2.8 & 2.2 & 4.7 & 6.9 & \\
\hline uid. 7711 & 2.6 & 2.5 & & 3.0 & $\cdots$ & & $\cdots$ & & \\
\hline uid. 7774 & 2.9 & 2.7 & & 2.6 & & & $\ldots$ & & \\
\hline$[\mathrm{Fe} \mathrm{XI}] 7890$ & 26.1 & 23.8 & & & & & & & \\
\hline
\end{tabular}




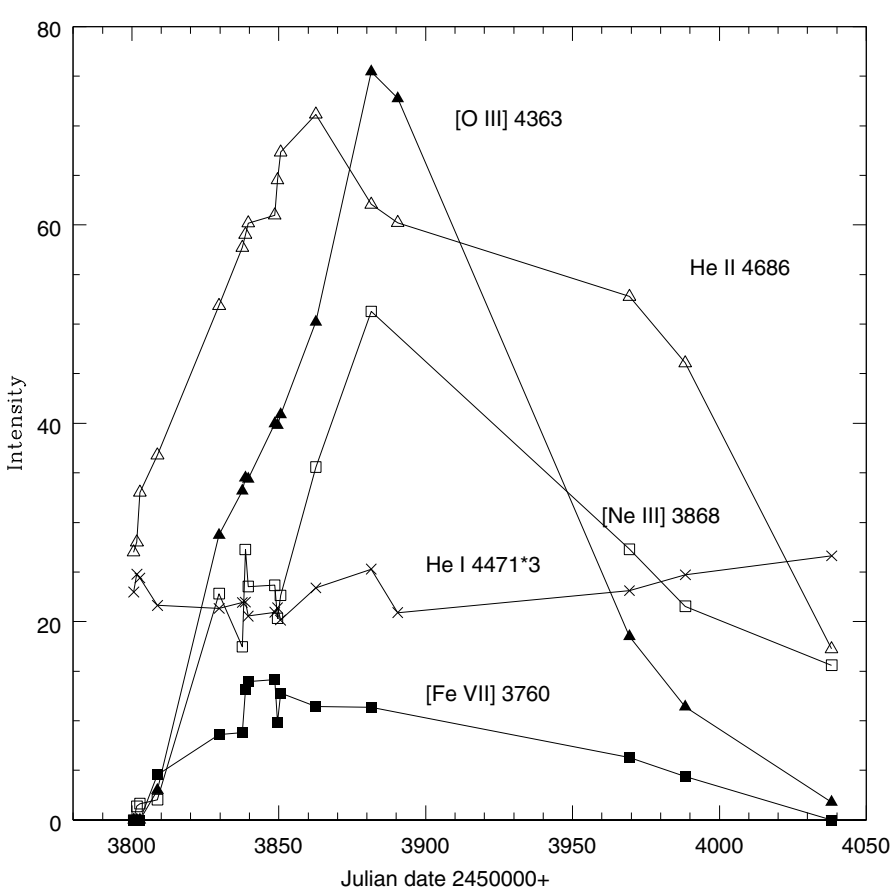

Fig. 45. Relative intensities, $\mathrm{H} \beta=100$, of selected emission lines.

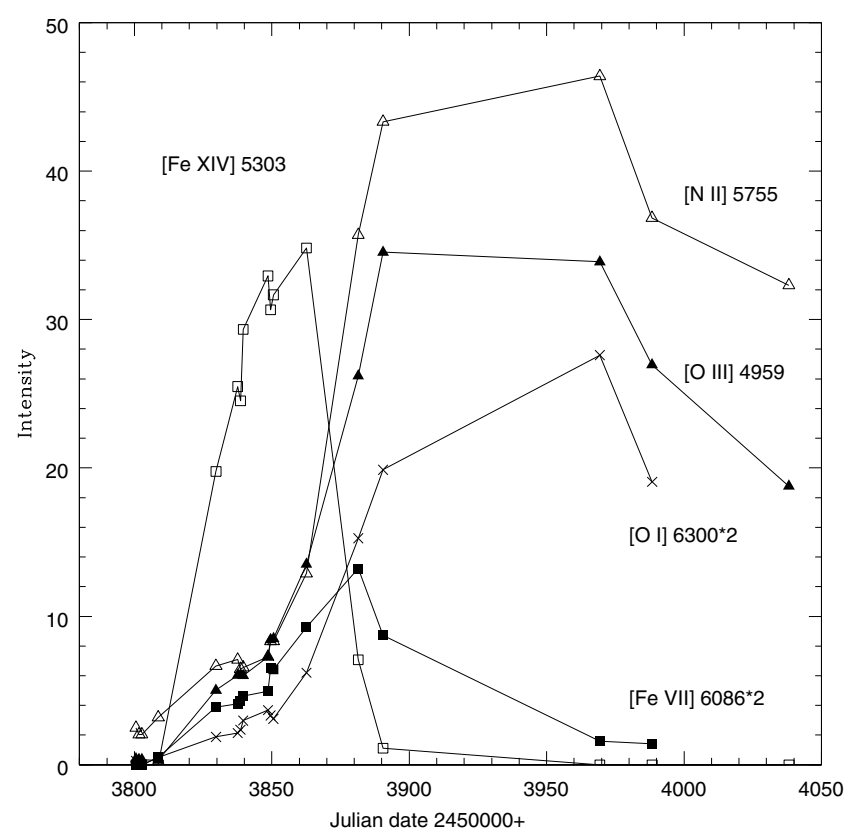

Fig. 46. Relative intensities, $\mathrm{H} \beta=100$, of selected emission lines.

strengthened, the Raman band at $6830 \AA$ disappeared and the line of O IV 6106 significantly faded. On the other hand, the intensities of the He II lines and most of the other lines did not vary much.

The so-called helium flash of classical novae occurs about 3.6 mag below maximum luminosity at which the emission lines of He I appear, then grow rapidly in intensity (McLaughlin 1960). Similar helium flashes were observed also in the historical outbursts of RS Oph (Rosino 1987). The present flare-up of He I occurred about 3.8 mag below maximum luminosity,

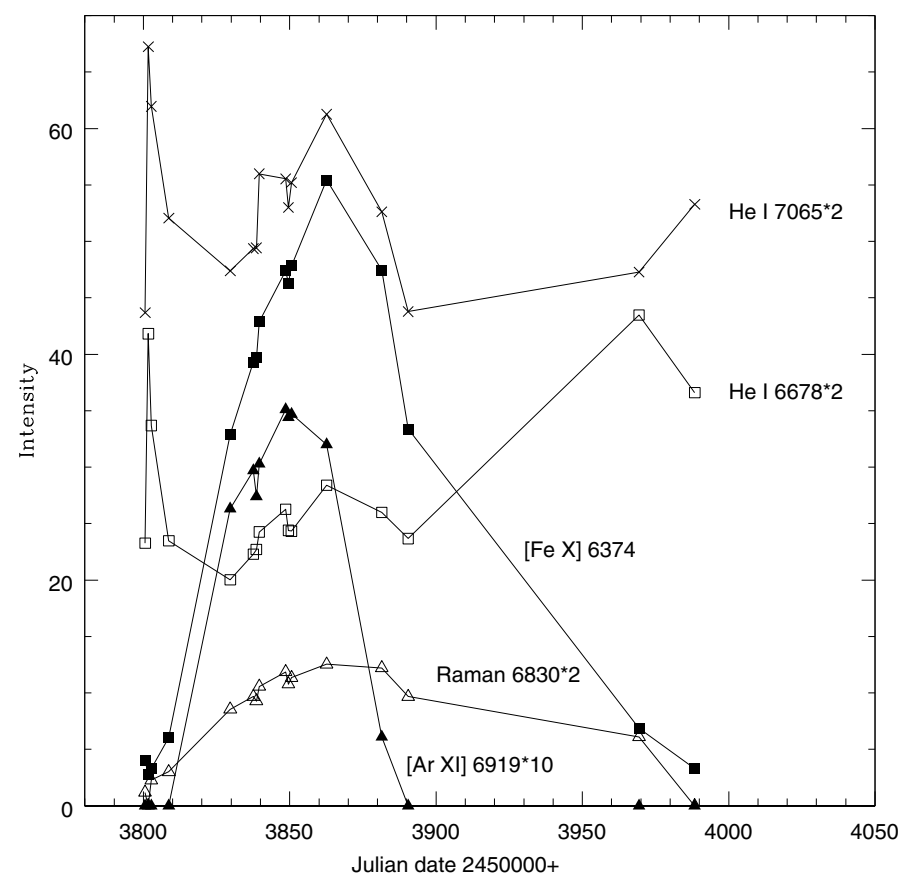

Fig. 47. Relative intensities, $\mathrm{H} \beta=100$, of selected emission lines.

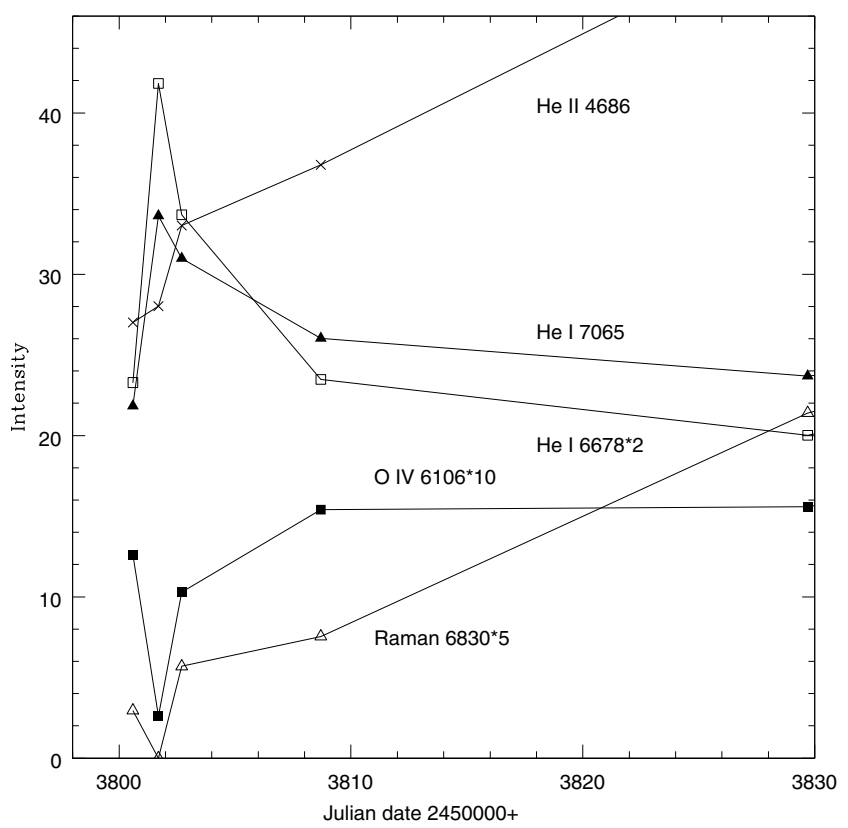

Fig. 48. Variations of the intensities of selected emission lines in the flare-up of He I lines on March 7: JD2453801.7.

where the photometric data presented in VSNET were used. There were, however, some serious differences between the helium flash of classical novae and the flare-up of He I lines of this object. For examples, the emission lines of He I did not appear on the flare-up, but were already prominent in February (Sect. 3.1), and the emission lines of He II were prominent when this phenomenon occurred. The duration of the flare-up was very short, and the He I lines began to fade the next day (Fig. 48, Table 2). 
The helium flash of classical novae occurs according to rising ionization level of ejecta. In contrast, the ionization level seems to have temporarily decreased in the flare-up of He I lines. Probably, the ionizing photons for He I (i.p.: $24.5 \mathrm{eV}$ ) increased and those for O IV (i.p.: $77.1 \mathrm{eV}$ ) decreased, meanwhile those for He II (i.p.: $54.2 \mathrm{eV}$ ) accidentally lay near the mirror point of the increasing and decreasing data. The flare-up of He I lines was possibly related to the oscillation of the pseudo-photosphere of the explosive object, which is usually observed in the transient stage of classical novae (McLaughlin 1960). Quantitative analyses are needed.

\subsection{Coronal emission lines}

The medium dispersion spectra obtained on March 14, day 29.4, showed no trace of coronal emission lines (Table 2). The coronal emission line [Fe XIV] 5303 was first detected on March 20: day 35.4 (Fig. 14). Most of the coronal lines reached their maximum intensities relative to $\mathrm{H} \beta$ around $70 \div 80$ days after the outburst, then faded rapidly (Figs. 46, 47 and Table 2), while [Fe X] 6374 faded more slowly (Fig. 47).

It is noteworthy that the intensities relative to $\mathrm{H} \beta$ of the coronal lines reached their maxima at stages earlier than those for [Fe VII] 5721 and 6086 (Figs. 46,47, Table 2). This result suggests that the coronal lines were not due to photo-ionization, because the temperature of the ionizing source is expected to have increased with time in this phase. The coronal lines were more probably due to the collision between the high velocity ejecta and the circumstellar envelope. The slow fading of the emission line of [Fe X] 6374 suggests that this line was due both to the photo-ionization and the collisional ionization.

Hachisu et al. (2007) collected the data of the flux of supersoft X-rays of RS Oph in the outburst obtained by the Swift XRT observations. They showed that the super-soft X-rays started to strengthen at about 30 days after the outburst, then an oscillation in intensity was seen until day 40 . The maximum flux was found at around day 50, then a slightly lower plateau continued until day 80 . The flux drastically declined between days 80 and 100 (Hachisu et al. 2007).

The intensities of the highly photo-ionized lines, e.g. He II, [Fe VII], and the Raman band at 6830, reached maxima in the period between days 71 and 102 (Figs. 45-47; Table 2), then decreased slowly until day after 200 . These variations were possibly related to the temperature of the ionizing source, namely, of the white dwarf in the system. The flux of the super-soft X-rays did not vary according to these variations, but varied nearly coincidentally with those of the coronal lines [Fe XIV] and [Ar XI] (Figs. 46, 47) . These phenomena suggest that the super-soft $\mathrm{X}$-rays were not due to the thermal radiation of the white dwarf, but were emitted in the collisional shock front.

When the coronal lines began to fade at about day 90, the forbidden lines of [O I], [N II], and [O III] started to strengthen rapidly (Fig. 46). Probably, the collisional shock front of the ejecta reached the outer boundary of the circumstellar envelope at that time and a free expansion started, which brought down the electron density of the ejecta.

\subsection{Unidentified emission lines}

As reported in Sect. 3.4, the emission line at $6106 \AA$ was prominent in our spectra (e.g., Fig. 32). This line is presented as an unidentified one at $6105.5 \AA$ in the catalog of Meinel et al. (1968). Dean \& Citters (1970) found this line in some spectra of the symbiotic star Z And, and identified it as [Cr I] 12F, 6106.2 A. On the other hand, Thackeray (1977) found the same line in his spectra of the symbiotic nova RR Tel and doubted its identification. Our results support Thackeray (1977), because no forbidden line of neutral metal was detected in our spectra. In our previous work of the 1985 outburst, we used a prismatic spectrograph whose observational error in wavelength was large, especially in the red region. Thus, we preliminarily identified it as [K IV] 1F, 6101.1 (Rosino \& Iijima 1987). This identification, however, is unlikely, because the observed wavelength in the present work does not agree with that of [K IV] 6101.1 , even if the largest observational error is adopted.

We estimated the laboratory wavelength of this line as $6106.2 \pm 0.3 \AA$. No acceptable candidate is found in the table of Moore (1959), but one possible identification is presented in the NIST atomic spectral database, that is O IV $4 \mathrm{~s} 2 \mathrm{P}^{0}-4 \mathrm{p} 2 \mathrm{~S}$, $6105.93 \AA$. This identification as the O IV line (i.p.: $77.1 \mathrm{eV}$ ) is consistent with the ionization level of the spectra, because when this line was first detected on March 6 (Fig. 10, Table 2), the emission lines of He II (i.p.: $54.2 \mathrm{eV}$ ) were prominent, but those of [Fe VII] (i.p.: $101 \mathrm{eV}$ ) were not seen yet. Unfortunately, the other lines of O IV in the optical region were hard to detect. For examples, O IV 9, 4798.3 was blended with Ti II 17, 4798.5 and [Fe II] 4F, 4798.3; O IV 11, 5362.4 was blended with Fe II 48, 5362.9 and [Fe II] 17F, 5362.1; and O IV 15, 4568 was blended with Ti II 60, 4568.3.

We detected a weak emission line at $6182.0 \pm 1 \AA$ in some spectra, whose intensities were roughly one fourth of those of the line at $6106 \AA$ (Figs. 18, 19, Table 2). This line is also presented as an unidentified one at $6181.2 \AA$ in the catalog of Meinel et al. (1968). Wallerstein \& Garnavich (1986) detected this line in some spectra of RS Oph on the 1985 outburst, and tentatively identified it as [Mn XIV] 6182.4. This identification, however, seems to be unlikely, because the line was detected also when the coronal lines were not seen, e.g., on March 14 (Table 2). Even though the observed wavelength is slightly different to the tabulated one, this line could be identified as O IV $4 \mathrm{~s} 2 \mathrm{P}^{0}-4 \mathrm{p} 2 \mathrm{~S}$, $6183.35 \AA$ in the NIST database.

We found two emission lines at 7711 and $7774 \AA$ in the spectrum on April 24 (Fig. 19). We are not able to determine the epoch of their appearance, because the spectra in March did not cover their spectral region. These lines were prominent in April (Table 2), then disappeared between May 7 and 25, as did most of the coronal emission lines (Table 2). Their laboratory wavelengths are estimated as $7711 \pm 2$ and $7774 \pm 2 \AA$, respectively. They seem to be coronal emission lines, but we are not able to find reasonable identifications.

A broad emission complex was seen at about $7322 \AA$ in some spectra. Its weak traces were seen in April (Figs. 18, 19), and it grew much in intensity on May 25 (Fig. 35) contemporaneously with the other non-coronal forbidden lines. At the same time, three peaks were observed of which the laboratory wavelengths were 7322,7328 , and $7340 \AA$, with errors of $\pm 2 \AA$. Emission lines of [O II] 2F, 7319 and 7330 are known in the spectra of novae and related objects, e.g., in RR Tel (Thackeray 1977). However, we hesitate to identify the former two lines as those of [O II], because the peak at 7322 was higher than that at 7328 in April, while the ratio was reversed on May 25. The emission line at $7322 \AA$ probably was a blend of [O II] 7319 and Fe II 73, 7320.7 (Moore 1959), and the latter was dominant when the emission at 7322 was stronger than that at 7328 in April and September (Table 2). One candidate for the line at $7340 \AA$ is [Cr IV] 1F, 7338, but another line of [Cr IV] of the 
same multiplet at 7390.6 ̊ (Moore 1959) was not detected. This emission line probably belonged to a non-coronal forbidden line, but further observations with higher dispersions are needed for identification.

\subsection{Raman band at $6830 \AA$}

The broad emission band at $6830 \AA$ was first noticed in the spectra of RS Oph in the 1933 outburst (Joy \& Swings 1945). Many highly ionized symbiotic stars have this emission band, but none of classical novae or planetary nebulae (Allen 1980). Schmid (1989) proposed that this emission band was due to Raman scattering of the O VI resonance line at $1031.9 \AA$ by neutral hydrogen. His model was confirmed by subsequent observations of symbiotic stars (Schmid et al. 1999 and references therein).

Our early spectra showed a weak but clear emission band at $6830 \AA$ when the emission lines of [Fe VII] were not seen (Fig. 10, Table 2). This result appears to be inconsistent with the model of Schmid (1989), because the ionization potential of O VI $(137.5 \mathrm{eV})$ is higher than that of [Fe VII] $(101 \mathrm{eV})$.

When the flare-up of He I lines occurred on March 7 (Sect. 4.1), the emission band at $6830 \AA$ and the O IV 6106 line faded contemporaneously (Fig. 48). This phenomenon suggests a close relation between the 6830 band and the O IV line. Some lines of O IV $3 \mathrm{~d}_{4} \mathrm{D}^{0}-4 \mathrm{f} 4 \mathrm{D}$ with wavelengths at 1033.07, $1033.37,1033.64 \AA$, etc. are given in the NIST database. Since high velocity gas motions were observed in the ejecta of RS Oph, these lines of O IV could have contributed to the Raman scattering. Our results suggest that the lines of O IV at about $1033 \AA$ play a certain role for the weak 6830 band under low ionization conditions.

When the emission band at 6830 became strong with the appearance of the emission lines of [Fe VII], its intensity did not correlate with that of O IV 6106. For example, the intensity of the 6830 band roughly tripled between March 14 and April 4, but that of O IV 6106 was nearly stable (Fig. 48, Table 2). The Raman scattering of O VI $1032 \AA$ proposed by Schmid (1989) seems to have become dominant at that time.

\section{Profiles of emission lines}

\subsection{Variations of the profiles}

Figures 49-51 show the serial variations of the profiles of $\mathrm{H} \beta$, He II 4686, and He I 5876. The height of each trace is normalized at unity, and the abscissas are heliocentric radial velocities in $\mathrm{km} \mathrm{s}^{-1}$.

As seen in the figures, the profiles changed much between February 19 and March 20. It seems that the red-shifted emission wings were dominant in the emissions on February 19, while the blue-shifted ones became stronger on March 20 and later. In the successive period, the emission wings faded with respect to the central components and the shifts of the wings decreased. The fading of the red-shifted wings was faster than those of the blueshifted ones.

\subsection{Gaussian decomposition}

Figures 52-54 show examples of decomposition in four Gaussian functions of $\mathrm{H} \beta$, He II 4686, and He I 5876 observed on March 20. In each figure the solid line is the observed profile, while the four components are shown by dotted lines: a central narrow component, blue- and red-shifted wings, and a

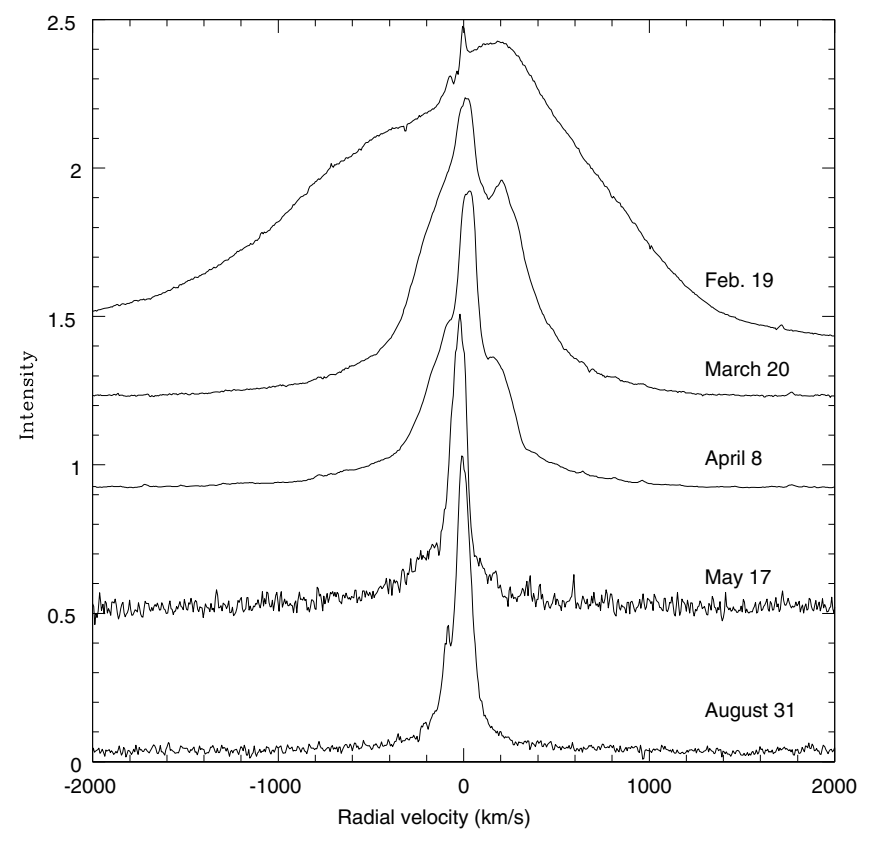

Fig. 49. Serial variation of the profile of $\mathrm{H} \beta$.

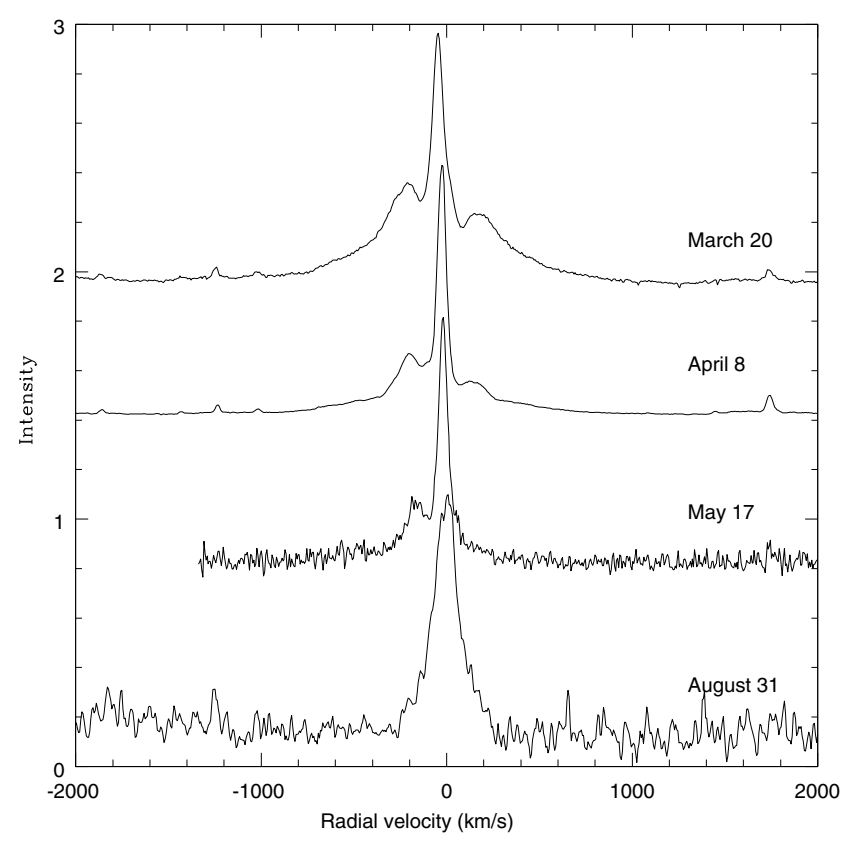

Fig. 50. Serial variation of the profile of He II 4686.

broad emission component. The fitted result is shown by a broken line.

The Gaussian decomposition is applied to the emission lines of $\mathrm{H} \beta$, He II 4686, and He I 5876 in the spectra obtained on March 20 and April 8. Table 3 presents the physical properties of the respective components. The errors in the radial velocities are about $\pm 1 \mathrm{~km} \mathrm{~s}^{-1}$.

The blue- or red-shifts of the wings were found in the range of about $\pm 200 \mathrm{~km} \mathrm{~s}^{-1}$, while the FWHMs of the broad emission components were an order of $1000 \mathrm{~km} \mathrm{~s}^{-1}$. The latter 


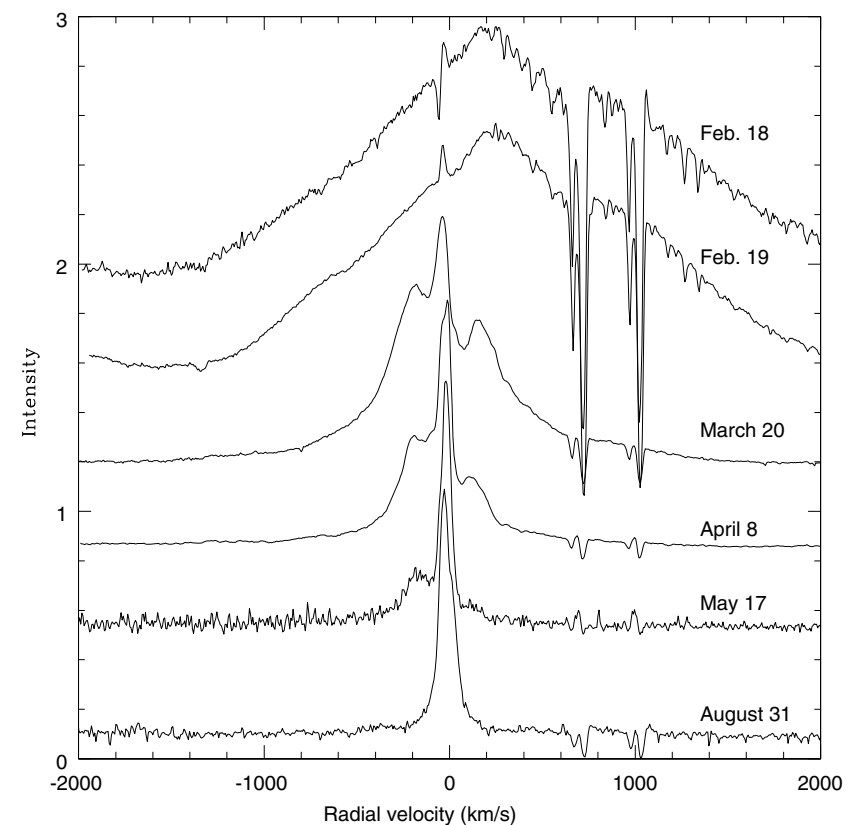

Fig. 51. Serial variation of the profile of He I 5876.

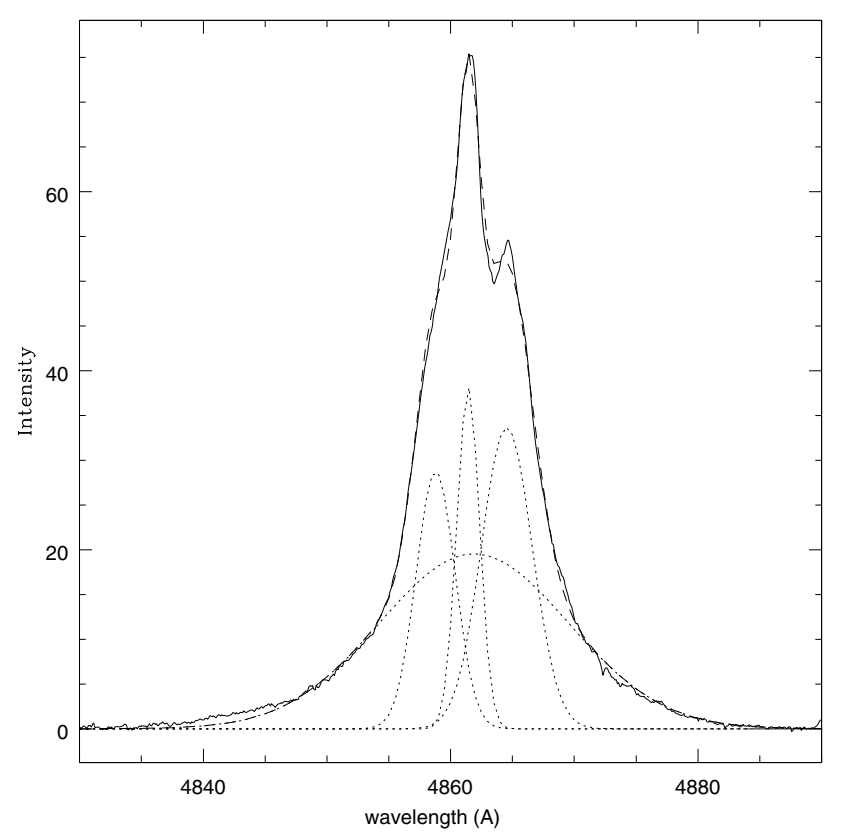

Fig. 52. Decomposition of $\mathrm{H} \beta$ on March 20 in four Gaussian functions.

velocities were of the same order as the expansion velocity of the ejecta expected from the model of O'Brien et al. (2006). The blue-shifts of the P Cygni type absorption components of X-ray spectra were also of the same order, $-1286 \pm 267 \mathrm{~km} \mathrm{~s}^{-1}$ on day 39.7 and $-771 \pm 65 \mathrm{~km} \mathrm{~s}^{-1}$ on day 66.9 (Ness et al. 2007). These velocities were related to the high velocity gas outflow of the outburst. On the other hand, our results show that about a half of the fluxes of the emission lines of H I, He I, and He II in these stages belonged to the stable circumstellar matter and the slowly moving gas clouds.

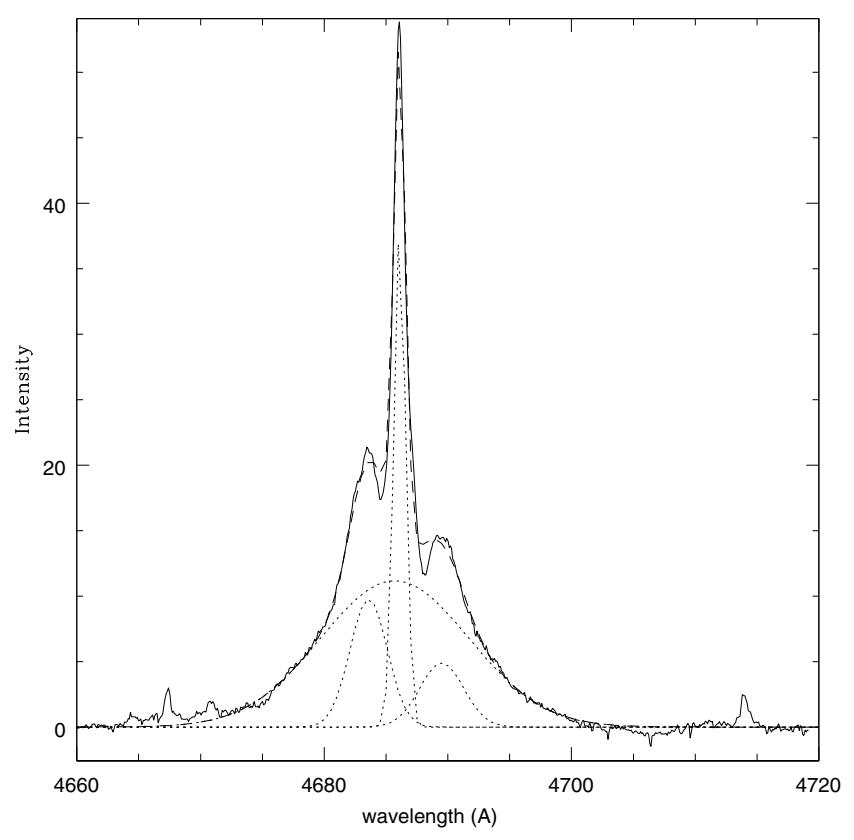

Fig. 53. Decomposition of He II 4686 on March 20 in four Gaussian functions.

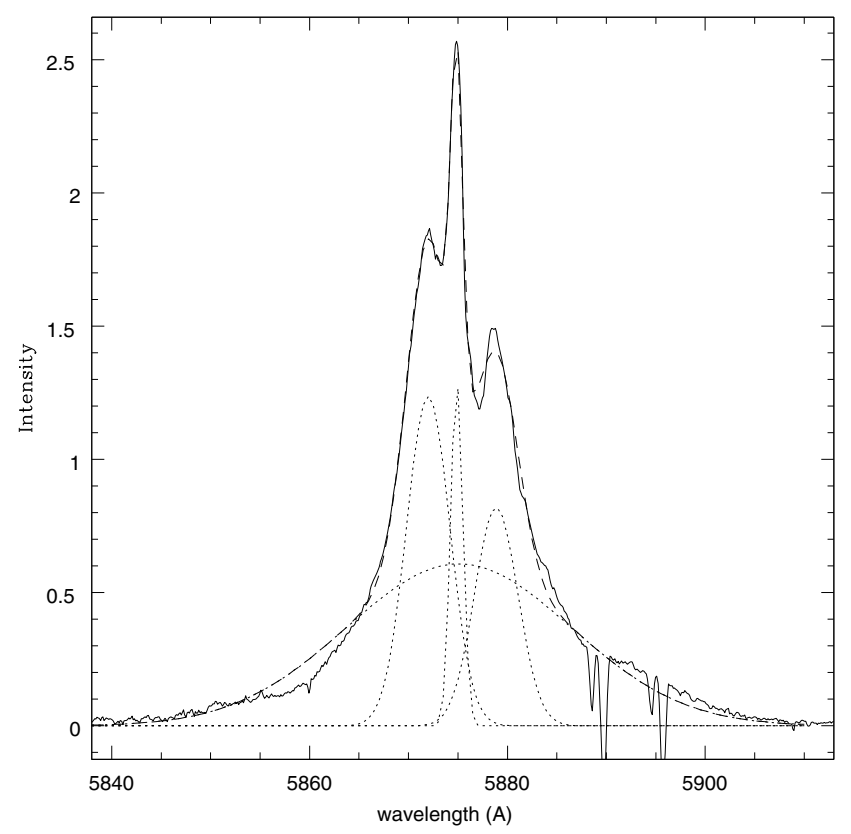

Fig. 54. Decomposition of He I 5876 on March 20 in four Gaussian functions.

Bode et al. (2007) obtained images of the ejecta with Hubble Space Telescope 155 days after the outburst. They found a peanut-shaped bipolar structure, and estimated that the extension of the ejecta along the equatorial regions was roughly one third of that along the polar regions. The expanding velocity of the circumstellar matter along the equatorial regions is expected to have been one third of that along the polar regions. Thus, the 
Table 3. Gaussian decomposition of prominent emission lines.

\begin{tabular}{|c|c|c|c|c|c|c|}
\hline \multirow{3}{*}{$\begin{array}{l}\text { Date } \\
\text { JD }\end{array}$} & \multirow{2}{*}{\multicolumn{3}{|c|}{$\begin{array}{c}\text { March } 20 \\
3814.7\end{array}$}} & \multirow{2}{*}{\multicolumn{3}{|c|}{$\begin{array}{l}\text { April } 8 \\
3833.6\end{array}$}} \\
\hline & & & & & & \\
\hline & r.v. & fwhm & flux & r.v. & fwhm & flux \\
\hline \multicolumn{7}{|c|}{$\mathrm{H} \beta$} \\
\hline central & +6 & 133 & 12 & +26 & 96 & 17 \\
\hline blue & -156 & 228 & 15 & -89 & 243 & 25 \\
\hline red & +198 & 298 & 23 & +170 & 186 & 15 \\
\hline broad & +35 & 1109 & 50 & +34 & 1060 & 43 \\
\hline \multicolumn{7}{|c|}{ He I 5876} \\
\hline central & -38 & 74 & 6 & -21 & 68 & 19 \\
\hline blue & -185 & 264 & 22 & -154 & 245 & 33 \\
\hline red & +166 & 264 & 15 & +133 & 145 & 9 \\
\hline broad & -27 & 1376 & 57 & -19 & 1129 & 39 \\
\hline \multicolumn{7}{|c|}{ He II 4686} \\
\hline central & +27 & 72 & 17 & +38 & 57 & 30 \\
\hline blue & -133 & 228 & 13 & -135 & 171 & 14 \\
\hline red & +244 & 269 & 8 & +213 & 101 & 2 \\
\hline broad & +1 & 906 & 62 & +40 & 784 & 54 \\
\hline
\end{tabular}

JD: Julian date + 2450000; r.v.: Heliocentric radial velocity in $\mathrm{km} \mathrm{s}^{-1}$ of line center; fwhm: Full width at half maximum; flux: Fraction of flux in $\%$.

radial velocities of the wings are comparable to the velocity of the circumstellar matter expanding along the equatorial regions.

\subsection{Splittings of narrow components}

The splittings of narrow emission components are not reported in any previous work as far as we know. Full widths at $90 \%$ of the height of the narrow emission components were measured to evaluate the splittings. When two peaks of the narrow components were observed, their separations were also measured. The results are presented in Table 4, where the numbers over the slash are the separations. The observational errors are about $\pm 1 \mathrm{~km} \mathrm{~s}^{-1}$.

The widths of the narrow emission components of [O III], [N II], Fe II, [Fe VII], and O IV 6106 were comparable to the resolution limit of our spectrograph, which is about $10 \mathrm{~km} \mathrm{~s}^{-1}$. The widths of $\mathrm{H} \mathrm{I}$, He I, and He II lines were of the order H I $>\mathrm{He}$ I $>\mathrm{He}$ II, and all of them decreased smoothly with time. On the other hand, the width of the coronal line [Fe XIV] 5303 was rather small on March 20, then increased to $70 \mathrm{~km} \mathrm{~s}^{-1}$ on April 8, and decreased to $22 \mathrm{~km} \mathrm{~s}^{-1}$ on May 17. The width of $\mathrm{H} \beta$ was larger than that of the coronal line [Fe XIV] 5303 in some spectra, but their profiles were somewhat different. The two narrow components were clearly separated in [Fe XIV] 5303 (Fig. 23), while slightly broader components were in contact in $\mathrm{H} \beta$ (Fig. 21). Thus, it is not sure whether the splittings of the coronal lines and those of the $\mathrm{H} \mathrm{I}$ and He I lines were due to the same mechanism.

The amounts of the splittings of the narrow components were much lower than the velocities related to the ejecta (Sect. 5.2). The splittings were possibly phenomena in the circumstellar matter, but the narrow emission components of [O III], [N II], $\mathrm{Fe}$ II, and [Fe VII], which belonged to the circumstellar matter, did not show any splitting. At the present time we have no idea of the cause of the splittings of the narrow components.

\section{Concluding remarks}

Detailed spectral evolution of RS Oph in the 2006 outburst is reported in this work. We found that the behaviors of the coronal emission lines were different from those of the other
Table 4. Full widths at $90 \%$ of the height of narrow emission components and the separations of two peaks in $\mathrm{km} \mathrm{s}^{-1}$.

\begin{tabular}{lrrr}
\hline \hline \multicolumn{1}{c}{ Date } & March 20 & April 8 & May 17 \\
& 3814.7 & 3833.6 & 3872.5 \\
\hline $\mathrm{He}$ II 4686 & 28 & 23 & 20 \\
$\mathrm{H} \beta$ & $37 / 86$ & $37 / 69$ & 31 \\
[O III] 5007 & 10 & 13 & \\
[Ni XIII] 5116 & & $49 / 74$ & \\
Fe II 5235 & 15 & 11 & \\
[Fe XIV] 5303 & 38 & $48 / 70$ & $16 / 22$ \\
Fe II 5316 & 14 & 13 & 9 \\
[Fe VII] 5721 & 11 & 15 & \\
[N II] 5755 & 12 & 13 & \\
He I 5876 & 47 & $33 / 41$ & 24 \\
O IV 6106 & & & 9 \\
[Fe X] 6374 & & & $15 / 22$ \\
\hline
\end{tabular}

JD: Julian date +2450000 ; the numbers over the slash are separations between two peaks.

forbidden lines (Sect. 4.2). The maximum intensities of most coronal emission lines relative to $\mathrm{H} \beta$ were found $70 \div 80$ days after the outburst. When the coronal lines started to fade about day 90 , the other forbidden lines began to strengthen. The variations in intensity of the coronal lines [Fe XIV] 5303 and [Ar XI] 6919 coincided with that of the flux of the super-soft X-rays reported by Hachisu et al. (2007). The coronal lines and the supersoft X-rays were probably emitted in the collisional shock front between the high velocity ejecta and the circumstellar envelope. The profiles of the prominent emission lines suggest that there was a rapidly expanding (of the order of $1000 \mathrm{~km} \mathrm{~s}^{-1}$ ), low density part and a slowly moving (of the order of $100 \mathrm{~km} \mathrm{~s}^{-1}$ ) high density part in the ejecta (Sect. 5.2), which were probably related to the expansion along the polar and the equatorial regions, respectively.

The emission lines at 6105.5 and $6181.2 \AA$ (Meinel et al. 1968) are identified as O IV 4s $2 \mathrm{P}^{0}-4 \mathrm{p} 2 \mathrm{~S}, 6105.9$ and 6183.4, respectively (Sect. 4.3). On the other hand, we found new unidentified, probably coronal, emission lines at 7711 and $7774 \AA$. A relationship between the Raman band at $6830 \AA$ and the lines of O IV $3 \mathrm{~d}_{4} \mathrm{D}^{0}-4 \mathrm{f} 4 \mathrm{D}$ at about $1033 \AA$ is suggested (Sect. 4.4).

The flare-up of the He I lines (Sect. 4.1) and the splitting of the narrow emission components (Sect. 5.2) are first reported in this work, and their causes are not understood yet. The numbers of days after the outburst are roughly 20 for the flare-up of the He I lines, 30 for the appearance of the coronal lines, 50 for the splitting of the narrow components, and 100 for the disappearance of the coronal lines. Detailed observations are needed of the next outburst.

Acknowledgements. We are grateful to Profs. I. Hachisu and M. Kato for the useful discussions and comments, and to Prof. R. Barbon for the careful reading of the manuscript and useful suggestions.

\section{References}

Allen, D. A. 1980, MNRAS, 190, 75

Barbon, R., Mammano, A., \& Rosino, L. 1969, in Non-Periodic Phenomena in Variable Stars, ed. L. Detre (Budapest: Academic Press), 257

Bode, M. F., O'Brien, T. J., Osborne, J. P., et al. 2006, ApJ, 652, 629

Bode, M. F., Harman, D. J., O'Brien, T. J., et al. 2007, ApJ, 665, L63

Chesneau, O., Nardetto, N., Millour, F., et al. 2007, A\&A, 464, 119

Das, R., Banerjee, D. P. K., \& Ashok, N. M. 2006, ApJ, 653, L141

Dean, C. A., \& van Citters, W. 1970, PASP, 82, 924 
Evans, A., Kerr, T., Yang, B., et al. 2007a, MNRAS, 374, L1

Evans, A., Woodward, C. E., Helton, L. A., et al. 2007b, ApJ, 663, L29

Hachisu, I., Kato, M., Kiyota, S., et al. 2006, ApJ, 651, L141

Hachisu, I., Kato, M., \& Luna, G. J. M. 2007, ApJ, 659, L153

Iijima, T. 2008, in RS Ophiuchi (2006) and the Recurrent Nova Phenomenon, ed. A. Evans, M. F. Bode, T. J. O’Brien, \& M.J. Darnley, ASP Conf. Ser., 401, 115

Joy, A. H., \& Swings, P. 1945, ApJ, 102, 353

Kerr, F. J., \& Westerhout, G. 1965, in Galactic Structure, ed. A. Blaauw \& M. Schmidt (The University of Chicago Press), 167

Lane, B. F., Sokoloski, J. L., Barry, R. K., et al. 2007, ApJ, 658, 520

McLaughlin, D. B. 1960, in Stellar Atmospheres, ed. J. L. Greenstein (The University of Chicago Press), 585

Meinel, A. B., Aveni, A. F., \& Stockton, M. W. 1968, Catalog of Emission Lines in Astrophysical Objects, Optical Sciences Center and Steward Observatory, The University of Arizona, Tucson
Moore, C. E. 1959, A Multiplet Table of Astrophysical Interest, US Dept. of Commerce, Office of Technical Services, Washington DC

Narumi, H., Hirosawa, K., Kanai, K., et al. 2006, IAU Circ. 8671

Ness, J.-U., Starrfield, S., Beardmore, A. P., et al. 2007, ApJ, 665, 1334

O'Brien, T. J., Bode, M. F., Porcas, R. W., et al. 2006, Nature, 442, 279

Osterbrock, D. E. 1989, Astrophysics of Gaseous Nebulae and Active Galactic Nuclei (Mill Valley, CA: University Science Books)

Rosino, L. 1987 in RS Ophiuchi (1985) and the Recurrent Nova Phenomenon, ed. M. F. Bode (Utrecht: VNU Science Press), 1

Rosino, L., \& Iijima, T. 1987 in RS Ophiuchi (1985) and the Recurrent Nova Phenomenon, ed. M.F. Bode (Utrecht: VNU Science Press), 27 Schmid, H. M. 1989, A\&A, 211, L31

Schmid, H. M., Krautter, J., Appemzeller, I., et al. 1999, A\&A, 348, 950

Thackeray, A. D. 1977, Mem. R. astr. Soc., 83, 1

Vaytet, N. M. H., O'Brien, T. J., \& Bode, M. F. 2007, ApJ, 665, 654

Wallerstein, G., \& Garnavich, P.M. 1986, PASP, 98, 875 
T. Iijima: Spectral evolution of RS Oph on the outburst in 2006, Online Material $p 1$

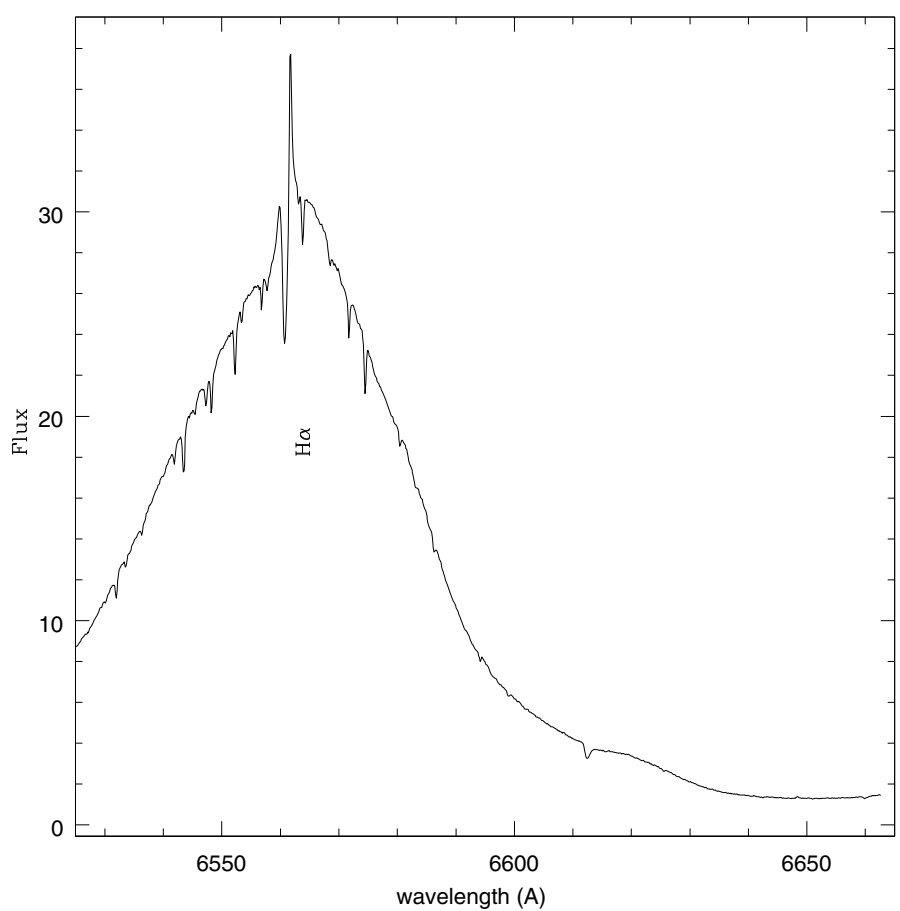

Fig. 1. Profile of $\mathrm{H} \alpha$ on Feb. 18. The unit of the ordinate is $10^{-11} \mathrm{erg} \mathrm{cm}^{-2} \mathrm{~s}^{-1} \AA^{-1}$.

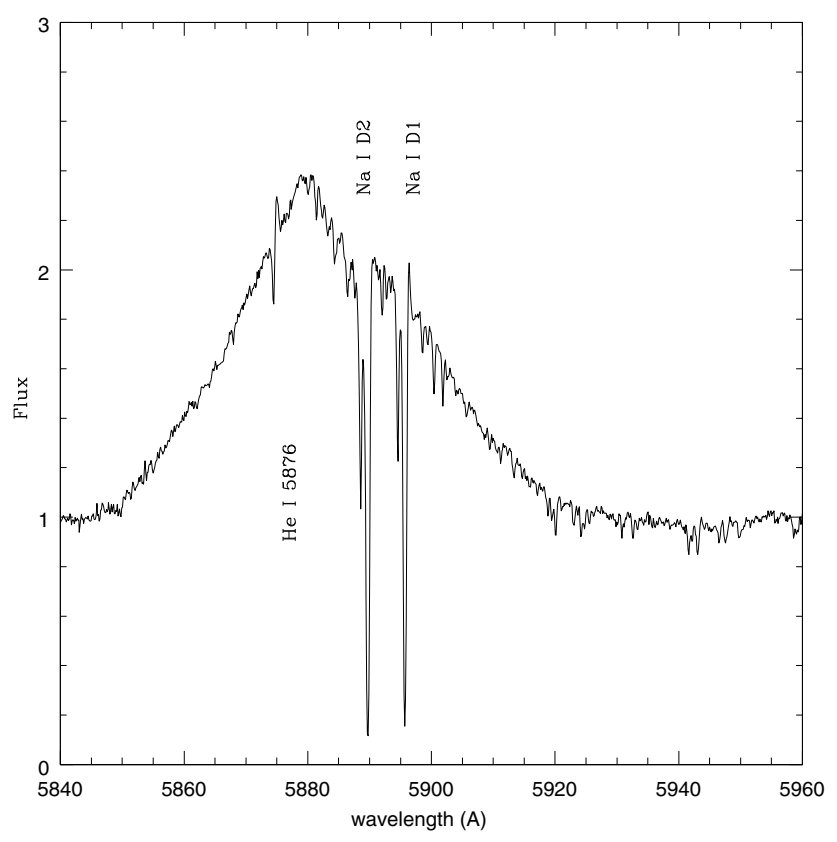

Fig. 2. Profile of He I 5876 on Feb. 18. The unit of the ordinate is $10^{-11} \mathrm{erg} \mathrm{cm}^{-2} \mathrm{~s}^{-1} \AA^{-1}$.
Table 1. Log of spectroscopic observations of RS Oph.

\begin{tabular}{|c|c|c|c|c|c|c|c|c|}
\hline $\begin{array}{l}\text { Date } \\
2006\end{array}$ & & $\overline{\mathrm{UT}}$ & JD & Days & $\begin{array}{r}\text { Exp. } \\
\text { sec }\end{array}$ & Inst. & $\begin{array}{c}\text { Range } \\
\mathrm{nm}\end{array}$ & Sky \\
\hline Feb. & 18 & $4: 28$ & 3784.7 & 5.4 & 300 & Ech & $516-686$ & \\
\hline Feb. & 18 & $4: 36$ & 3784.7 & 5.4 & 600 & & " & \\
\hline Feb. & 19 & 4:37 & 3785.7 & 6.4 & 600 & " & $420-596$ & \\
\hline Feb. & 19 & $4: 50$ & 3785.7 & 6.4 & 600 & " & " & \\
\hline Feb. & 19 & 5:04 & 3785.7 & 6.4 & 600 & " & " & \\
\hline March & 6 & $3: 11$ & 3800.6 & 21.3 & 600 & $\mathrm{~B} \& \mathrm{C}$ & $360-595$ & \\
\hline March & 6 & $3: 25$ & 3800.6 & 21.3 & 1200 & " & & \\
\hline March & 6 & 4:16 & 3800.7 & 21.4 & 60 & " & $500-735$ & \\
\hline March & 6 & $4: 19$ & 3800.7 & 21.4 & 300 & " & " & \\
\hline March & 6 & 4:26 & 3800.7 & 21.4 & 600 & " & $"$ & \\
\hline March & 7 & $3: 39$ & 3801.6 & 22.3 & 600 & " & $350-583$ & \\
\hline March & 7 & $3: 53$ & 3801.7 & 22.4 & 1200 & " & & \\
\hline March & 7 & $4: 51$ & 3801.7 & 22.4 & 300 & " & $520-757$ & \\
\hline March & 7 & $4: 57$ & 3801.7 & 22.4 & 60 & " & 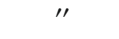 & \\
\hline March & 7 & $4: 59$ & 3801.7 & 22.4 & 30 & " & " & \\
\hline March & 8 & $3: 49$ & 3802.7 & 23.4 & 300 & " & $360-590$ & cloud \\
\hline March & 8 & 3:57 & 3802.7 & 23.4 & 1200 & " & & clou \\
\hline March & 8 & $4: 48$ & 3802.7 & 23.4 & 300 & " & $530-765$ & clou \\
\hline March & 14 & $3: 40$ & 3808.7 & 29.4 & 600 & " & $350-576$ & \\
\hline March & 14 & $3: 53$ & 3808.7 & 29.4 & 1800 & " & & \\
\hline March & 14 & $4: 53$ & 3808.7 & 29.4 & 300 & " & $530-765$ & \\
\hline March & 14 & $4: 59$ & 3808.7 & 29.4 & 60 & " & & \\
\hline March & 20 & $3: 32$ & 3814.7 & 35.4 & 600 & Ech & $420-596$ & \\
\hline March & 20 & $3: 46$ & 3814.7 & 35.4 & 600 & 1 & & \\
\hline April & 4 & $2: 32$ & 3829.6 & 50.3 & 600 & $\mathrm{~B} \& \mathrm{C}$ & $350-587$ & \\
\hline April & 4 & $2: 45$ & 3829.6 & 50.3 & 1800 & " & & \\
\hline April & 4 & $3: 58$ & 3829.7 & 50.4 & 600 & " & $535-775$ & \\
\hline April & 4 & 4:10 & 3829.7 & 50.4 & 120 & " & & \\
\hline April & 8 & $2: 42$ & 3833.6 & 54.3 & 1200 & Ech & $427-596$ & \\
\hline April & 8 & 3:03 & 3833.6 & 54.3 & 1200 & & & \\
\hline April & 8 & $3: 27$ & 3833.7 & 54.4 & 1200 & " & " & \\
\hline April & 12 & $1: 32$ & 3837.6 & 58.3 & 1800 & $\mathrm{~B} \& \mathrm{C}$ & $345-580$ & \\
\hline April & 12 & $2: 37$ & 3837.6 & 58.3 & 300 & II & $560-798$ & \\
\hline April & 12 & $2: 44$ & 3837.6 & 58.3 & 120 & " & & \\
\hline April & 12 & $2: 49$ & 3837.6 & 58.3 & 900 & " & $" 1$ & \\
\hline April & 13 & $1: 13$ & 3838.6 & 59.3 & 120 & " & $560-798$ & \\
\hline April & 13 & $1: 17$ & 3838.6 & 59.3 & 900 & " & & \\
\hline April & 13 & $1: 40$ & 3838.6 & 59.3 & 1800 & " & $360-600$ & \\
\hline April & 14 & $2: 15$ & 3839.6 & 60.3 & 60 & B\&C3 & $350-815$ & \\
\hline April & 14 & $2: 18$ & 3839.6 & 60.3 & 600 & 11 & & \\
\hline April & 23 & $1: 56$ & 3848.6 & 69.3 & 60 & " & $340-800$ & \\
\hline April & 23 & $1: 59$ & 3848.6 & 69.3 & 300 & " & & \\
\hline April & 23 & 2:08 & 3848.6 & 69.3 & 1200 & " & " & \\
\hline April & 24 & 2:07 & 3849.6 & 70.3 & 2400 & $\mathrm{~B} \& \mathrm{C}$ & $365-603$ & clou \\
\hline April & 24 & $3: 12$ & 3849.6 & 70.3 & 120 & " & $550-790$ & clot \\
\hline April & 24 & $3: 17$ & 3849.6 & 70.3 & 600 & " & & clo \\
\hline April & 25 & $1: 28$ & 3850.6 & 71.3 & 180 & " & $525-760$ & \\
\hline April & 25 & $1: 33$ & 3850.6 & 71.3 & 900 & " & & \\
\hline April & 25 & $2: 25$ & 3850.6 & 71.3 & 1800 & " & $360-593$ & \\
\hline May & 7 & $1: 44$ & 3862.6 & 83.3 & 2400 & " & $345-583$ & \\
\hline May & 7 & $2: 47$ & 3862.6 & 83.3 & 300 & " & $542-783$ & \\
\hline May & 7 & $2: 54$ & 3862.6 & 83.3 & 1200 & " & & \\
\hline May & 17 & 0:00 & 3872.5 & 93.2 & 1200 & Ech & $472-647$ & \\
\hline May & 17 & $0: 22$ & 3872.5 & 93.2 & 1200 & & & \\
\hline May & 17 & $0: 46$ & 3872.5 & 93.2 & 1200 & 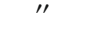 & " & \\
\hline May & 23 & $1: 28$ & 3878.6 & 99.3 & 3000 & $\mathrm{~B} \& \mathrm{C}$ & $340-575$ & \\
\hline May & 23 & $2: 45$ & 3878.6 & 99.3 & 300 & " & $525-760$ & \\
\hline May & 25 & 23:41 & 3881.5 & 102.2 & 300 & " & $540-780$ & \\
\hline May & 25 & 23:49 & 3881.5 & 102.2 & 1200 & " & " & \\
\hline May & 26 & $0: 53$ & 3881.6 & 102.3 & 3000 & " & $350-588$ & \\
\hline June & 3 & 23:00 & 3890.5 & 111.2 & 3600 & " & $340-574$ & clou \\
\hline June & 4 & $0: 42$ & 3890.5 & 111.2 & 600 & " & $525-765$ & cloudy \\
\hline June & 4 & $0: 55$ & 3890.5 & 111.2 & 1800 & " & & cloudy \\
\hline June & 10 & $0: 55$ & 3896.5 & 117.2 & 1200 & Ech & $472-647$ & \\
\hline June & 10 & $1: 17$ & 3896.6 & 117.3 & 1200 & & & \\
\hline
\end{tabular}


Table 1. continued.

\begin{tabular}{|c|c|c|c|c|c|c|c|}
\hline $\begin{array}{l}\overline{\text { Date }} \\
2006\end{array}$ & & $\overline{\mathrm{UT}}$ & $\overline{\mathrm{JD}}$ & $\overline{\text { Days }}$ & $\begin{array}{r}\text { Exp. } \\
\text { sec }\end{array}$ & $\overline{\text { Inst. }}$ & $\begin{array}{cc}\begin{array}{c}\text { Range } \\
\text { nm }\end{array} & \text { sky } \\
\end{array}$ \\
\hline June & 10 & $1: 42$ & 3896.6 & 117.3 & 1200 & & \\
\hline August & 7 & $21: 42$ & 3955.4 & 176.1 & 3000 & & \\
\hline August & 21 & 21:09 & 3969.4 & 190.1 & 600 & $\mathrm{~B} \& \mathrm{C} 3$ & $330-792$ \\
\hline August & 21 & $21: 22$ & 3969.4 & 190.1 & 1800 & " & "I \\
\hline August & 30 & 20:39 & 3978.4 & 199.1 & 2400 & Ech & $427-596$ \\
\hline August & 31 & $19: 49$ & & 200.1 & 3000 & & \\
\hline pt. & 1 & 19:48 & 39 & 201.1 & 1800 & " & $517-686$ \\
\hline Sept. & 5 & 20:17 & & 205.1 & 1800 & " & -569 \\
\hline Sep & 9 & $20: 13$ & & 209.1 & 3000 & $B \& C$ & 588 \\
\hline Sep & 12 & 20: & $39 c$ & 212.0 & 600 & 11 & $505-745$ \\
\hline Sept. & 12 & $20: 30$ & $39 c$ & 212.1 & 1800 & " & " \\
\hline Oct. & 29 & $17: 22$ & 4038.2 & 258.9 & 1800 & & $353-590$ \\
\hline
\end{tabular}

UT: Universal time at start of exposure; JD: Julian date 2450000 ; days: Number of days from maximum luminosity: JD2453779.3; B\&C3: A grating 300 lines $\mathrm{mm}^{-1}$ was used.

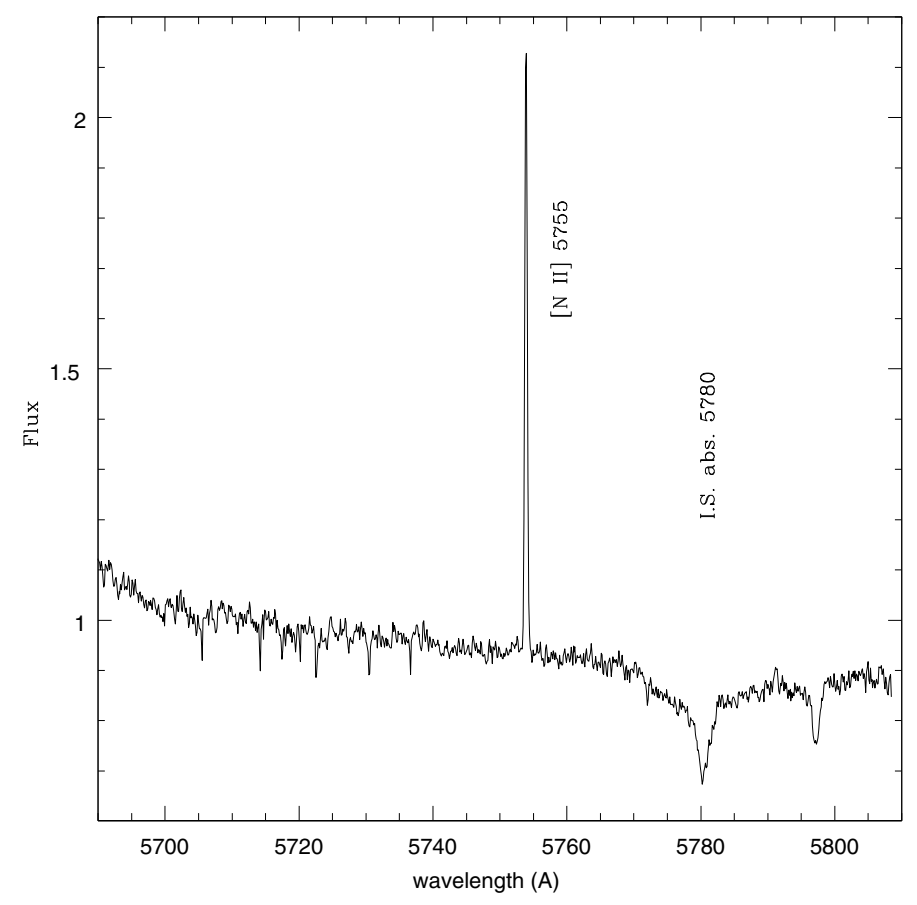

Fig. 3. Profile of [N II] 5755 on Feb. 18. The unit of the ordinate is $10^{-11} \mathrm{erg} \mathrm{cm}^{-2} \mathrm{~s}^{-1} \AA^{-1}$.

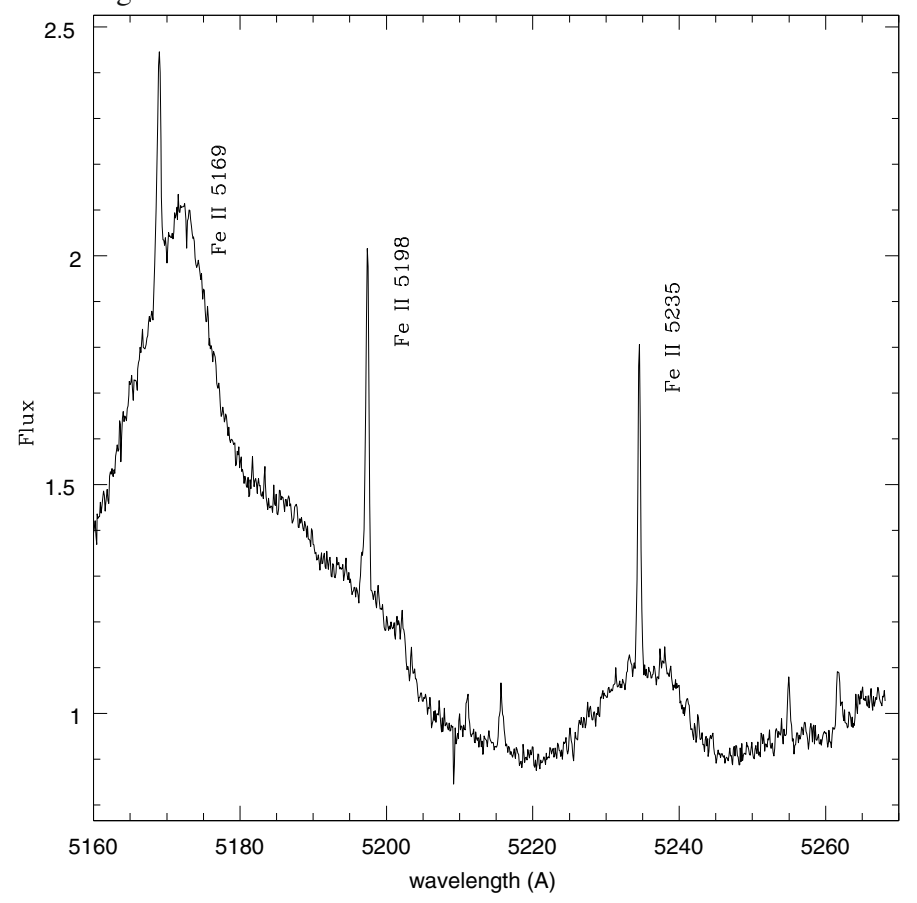

Fig. 4. Profiles of Fe II lines on Feb. 18. The unit of the ordinate is $10^{-11}$ $\operatorname{erg~cm}{ }^{-2} \mathrm{~s}^{-1} \AA^{-1}$. 
T. Iijima: Spectral evolution of RS Oph on the outburst in 2006, Online Material p 3

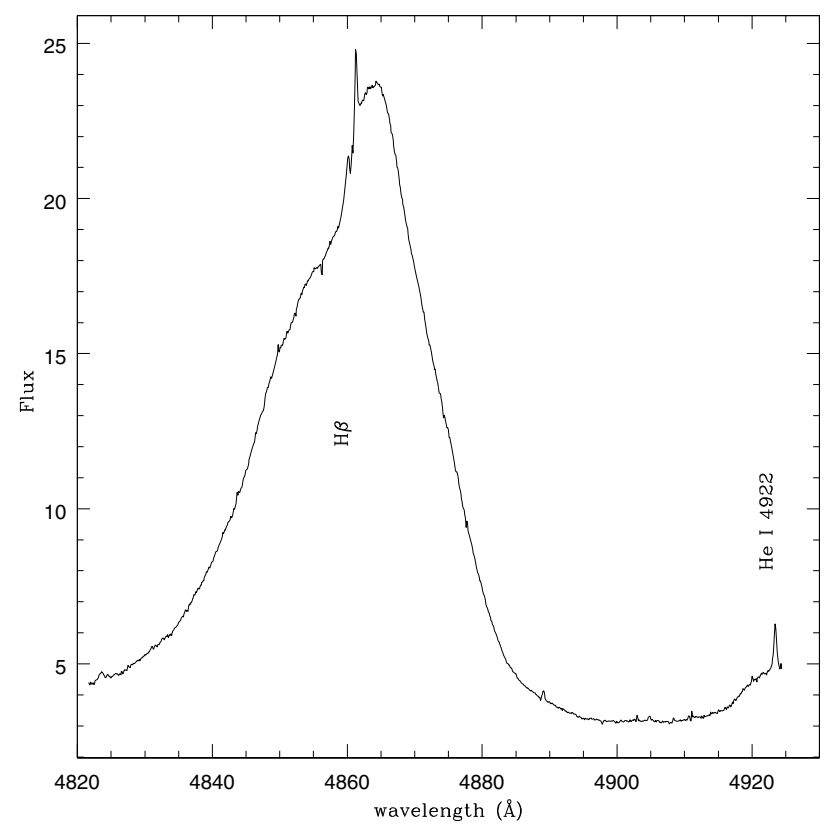

Fig. 5. Profile of $\mathrm{H} \beta$ on Feb. 19. The unit of the ordinate is $10^{-11} \mathrm{erg} \mathrm{cm}^{-2} \mathrm{~s}^{-1} \AA^{-1}$.

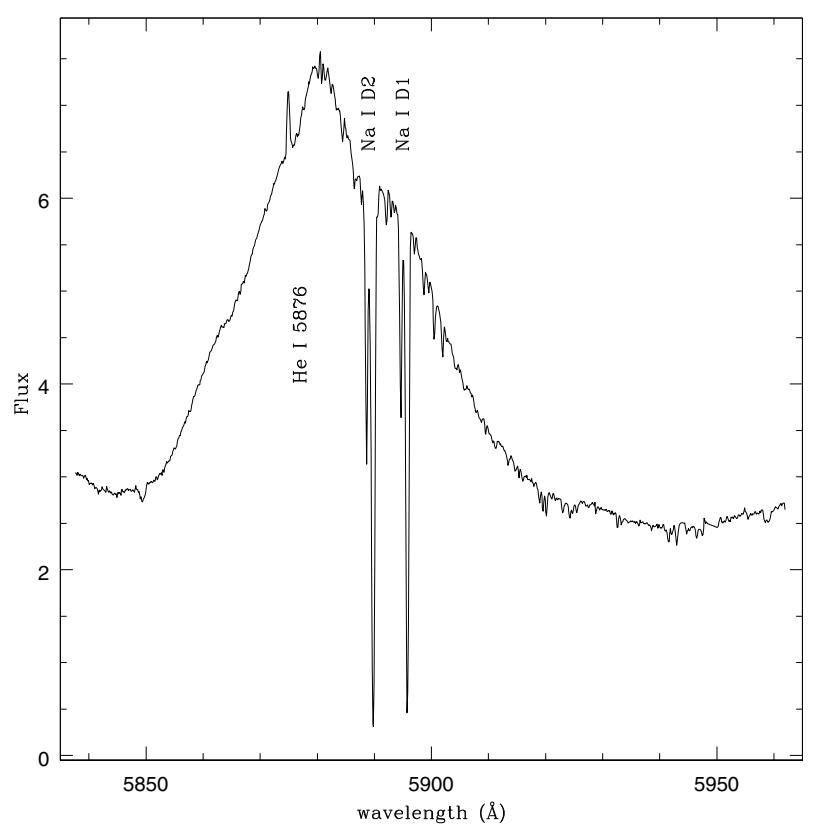

Fig. 6. Profile of He I 5876 on Feb. 19. The unit of the ordinate is $10^{-11} \mathrm{erg} \mathrm{cm}^{-2} \mathrm{~s}^{-1} \AA^{-1}$.

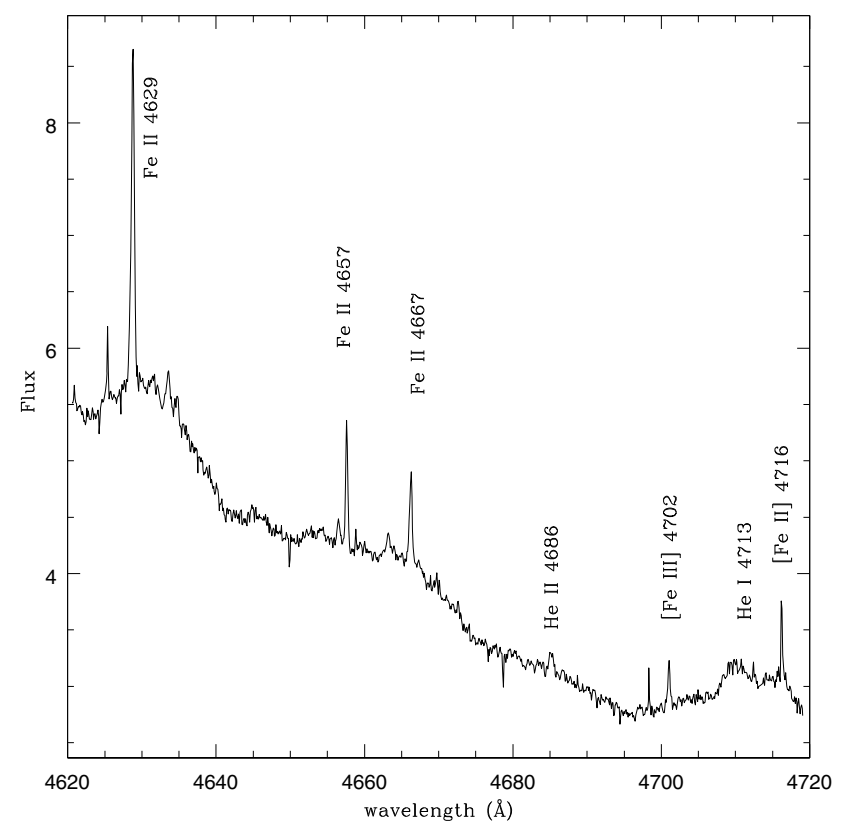

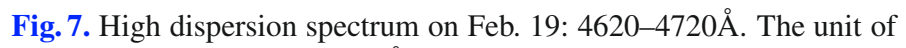
ordinate is $10^{-11} \mathrm{erg} \mathrm{cm}^{-2} \mathrm{~s}^{-1} \AA^{-1}$.

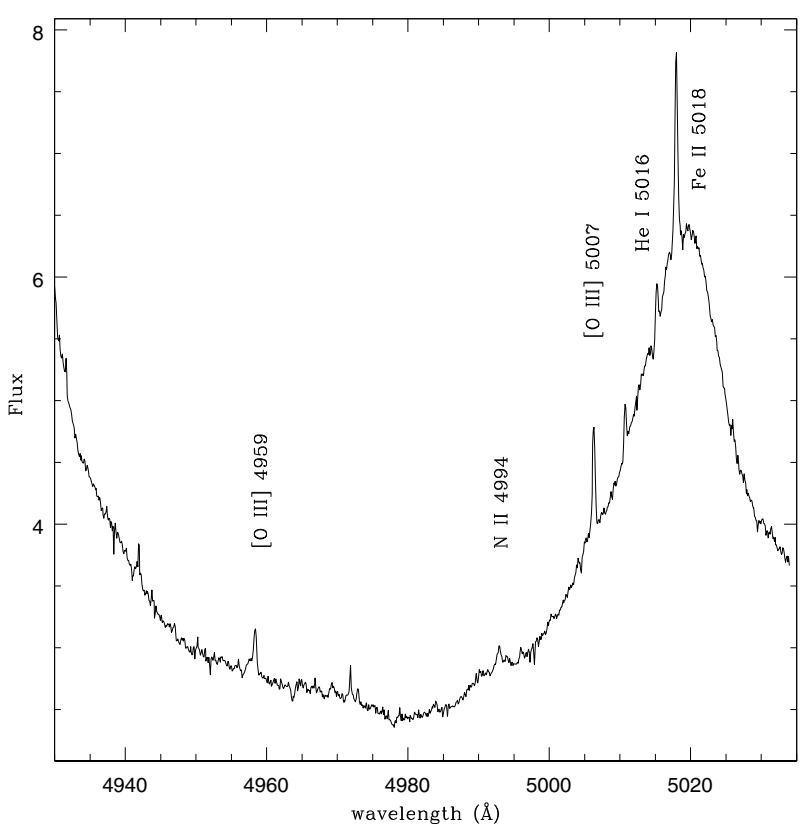

Fig. 8. High dispersion spectrum on Feb. 19: 4930-5030尺. The unit of ordinate is $10^{-11} \mathrm{erg} \mathrm{cm}^{-2} \mathrm{~s}^{-1} \AA^{-1}$. 
T. Iijima: Spectral evolution of RS Oph on the outburst in 2006, Online Material p 4

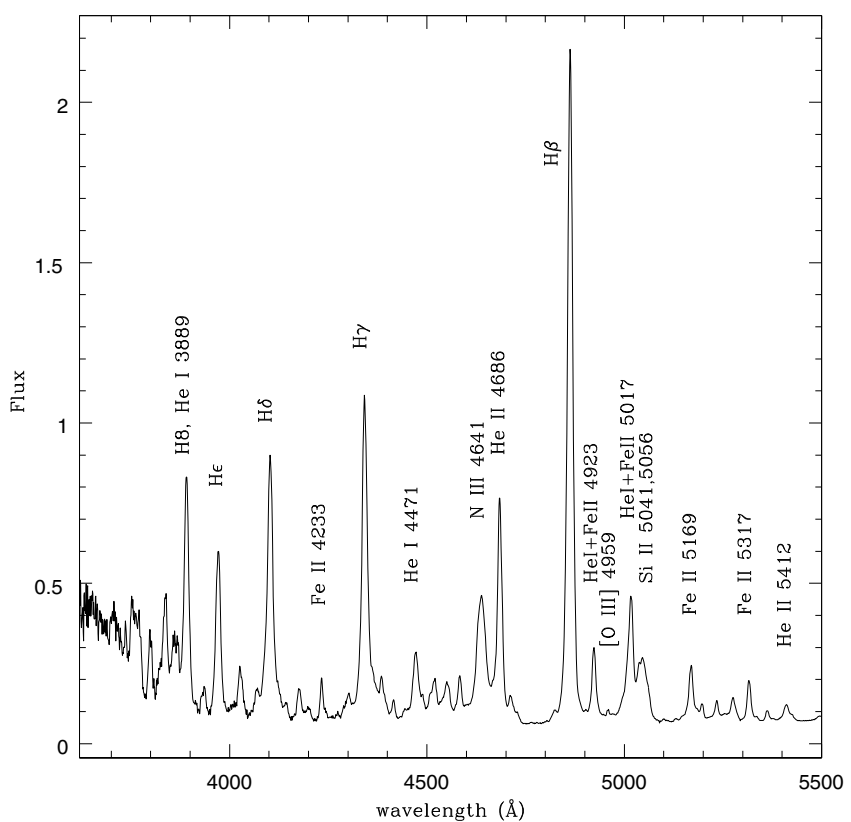

Fig. 9. Medium dispersion blue spectrum on March 6. The unit of ordinate is $10^{-11} \mathrm{erg} \mathrm{cm}^{-2} \mathrm{~s}^{-1} \AA^{-1}$.

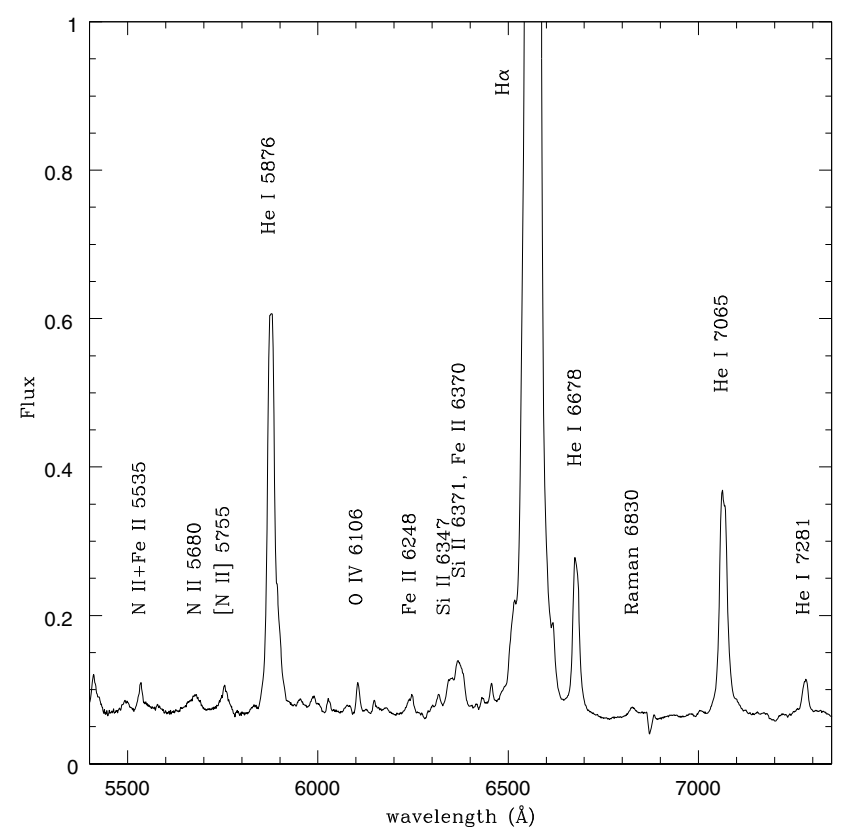

Fig. 10. Medium dispersion red spectrum on March 6. The unit of ordinate is $10^{-11} \mathrm{erg} \mathrm{cm}^{-2} \mathrm{~s}^{-1} \AA^{-1}$.

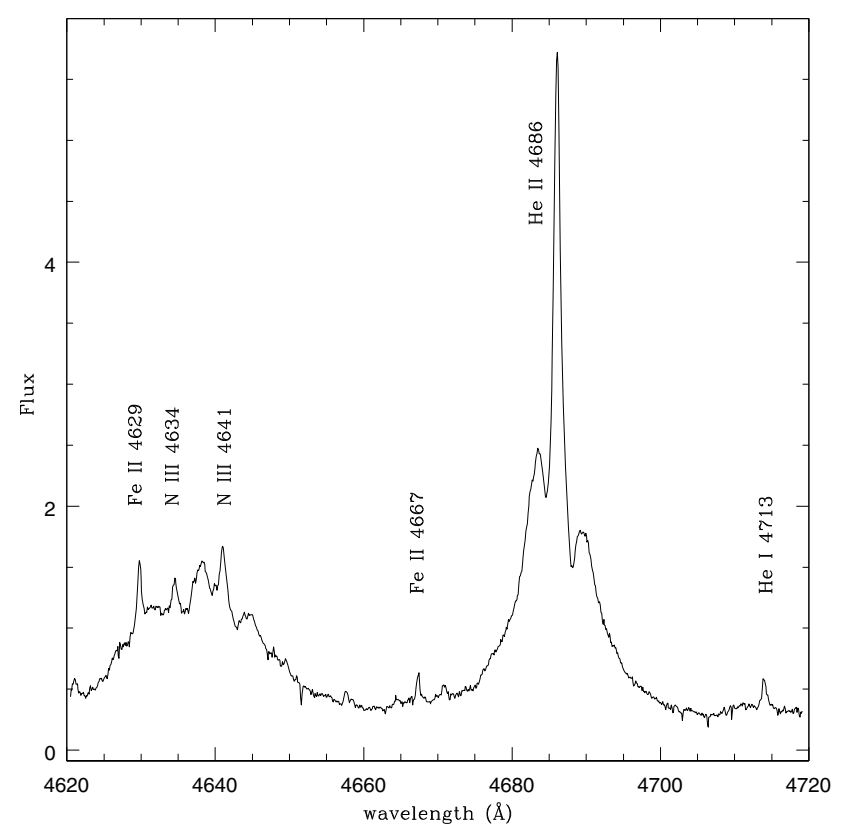

Fig. 11. High dispersion spectrum on March 20: 4620-4720 A. The unit of ordinate is $10^{-12} \mathrm{erg} \mathrm{cm}^{-2} \mathrm{~s}^{-1} \AA^{-1}$.

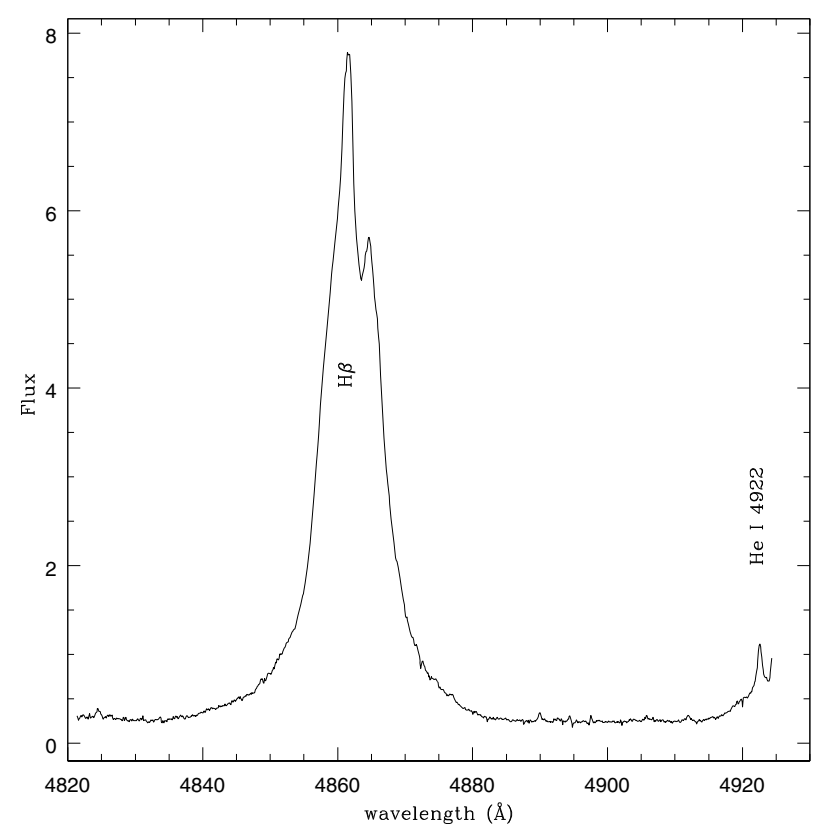

Fig. 12. Profile of $\mathrm{H} \beta$ on March 20. The unit of ordinate is $10^{-12} \mathrm{erg} \mathrm{cm}^{-2} \mathrm{~s}^{-1} \AA^{-1}$. 
T. Iijima: Spectral evolution of RS Oph on the outburst in 2006, Online Material p 5

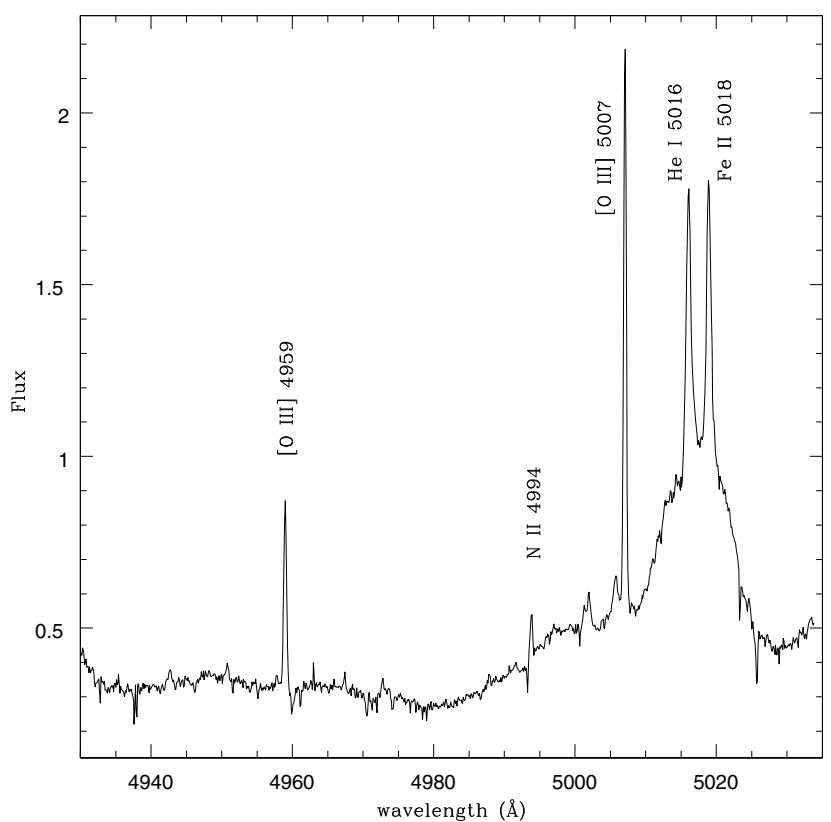

Fig. 13. High dispersion spectrum on March 20: 4930-5030 . The unit of ordinate is $10^{-12} \mathrm{erg} \mathrm{cm}^{-2} \mathrm{~s}^{-1} \AA^{-1}$.

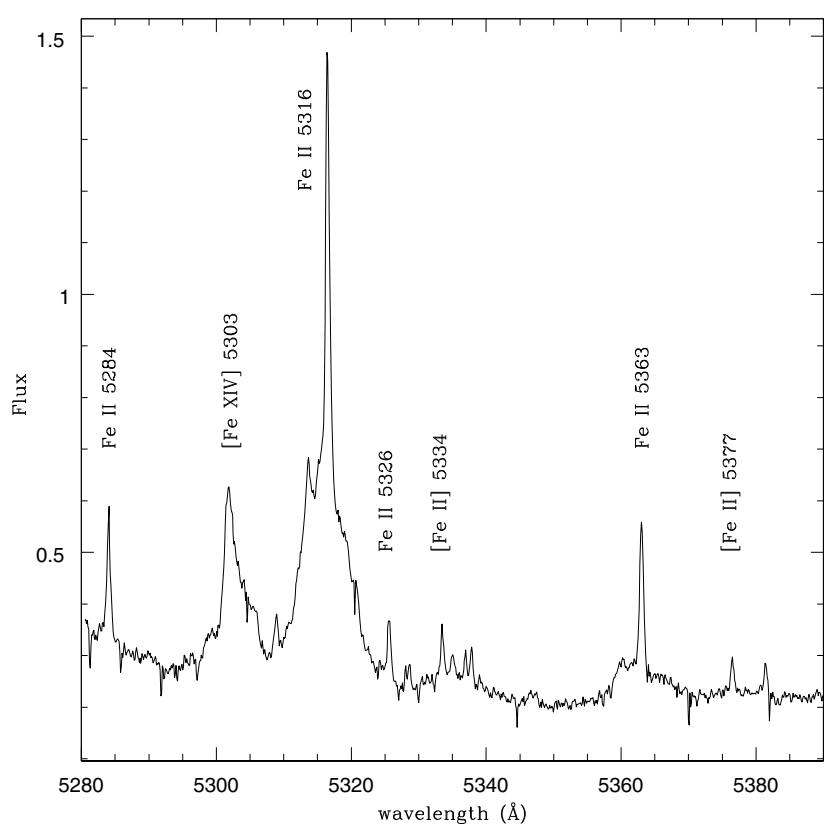

Fig. 14. High dispersion spectrum on March 20: 5280-5390A. The unit of ordinate is $10^{-12} \mathrm{erg} \mathrm{cm}^{-2} \mathrm{~s}^{-1} \AA^{-1}$.

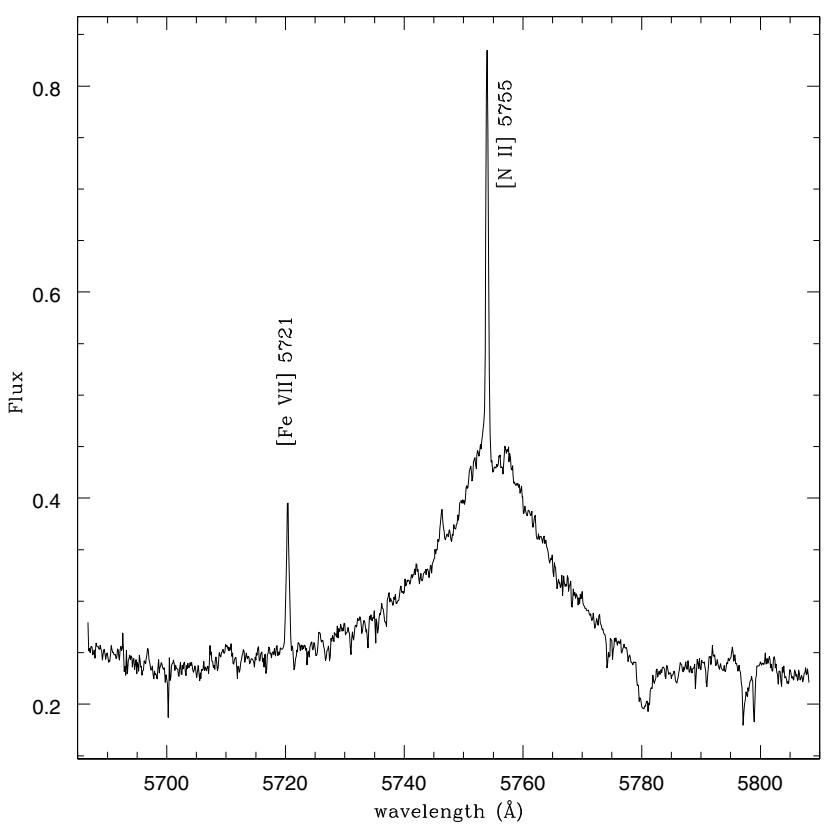

Fig. 15. High dispersion spectrum on March 20: 5690-5810 $\AA$. The unit of ordinate is $10^{-12} \mathrm{erg} \mathrm{cm}^{-2} \mathrm{~s}^{-1} \AA^{-1}$.

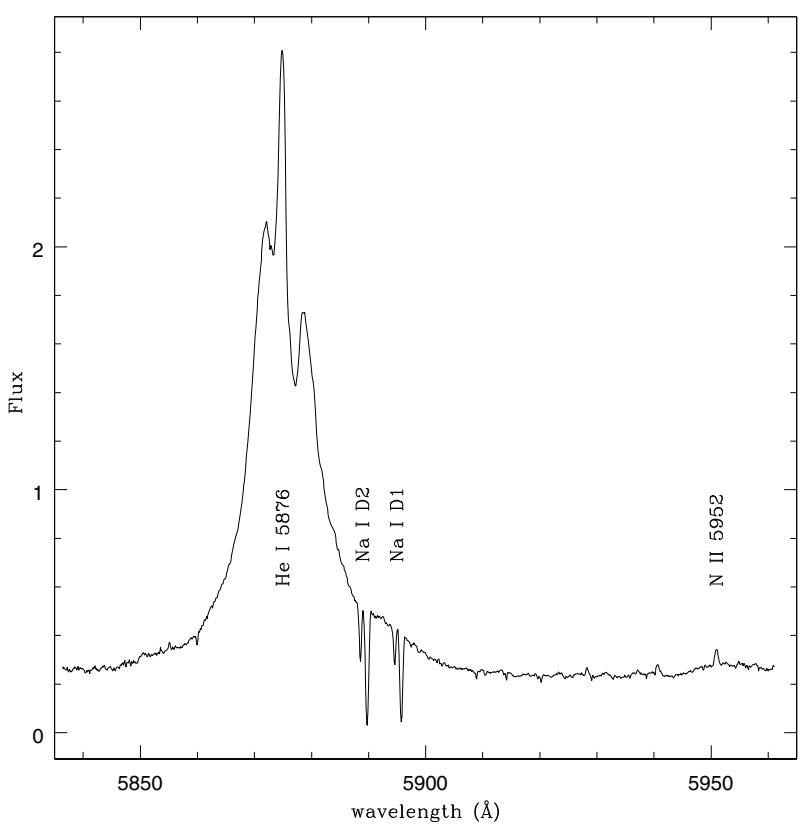

Fig. 16. Profile of He I 5876 on March 20. The unit of ordinate is $10^{-12} \mathrm{erg} \mathrm{cm}^{-2} \mathrm{~s}^{-1} \AA^{-1}$. 
T. Iijima: Spectral evolution of RS Oph on the outburst in 2006, Online Material p 6

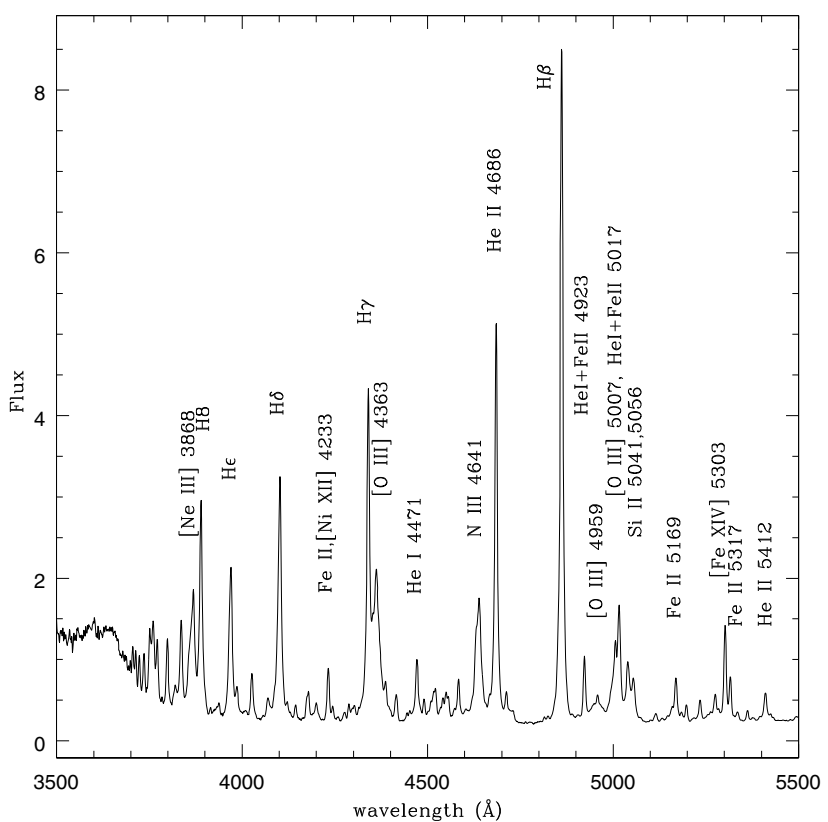

Fig. 17. Medium dispersion blue spectrum on April 4. The unit of ordinate is $10^{-12} \mathrm{erg} \mathrm{cm}^{-2} \mathrm{~s}^{-1} \AA^{-1}$.

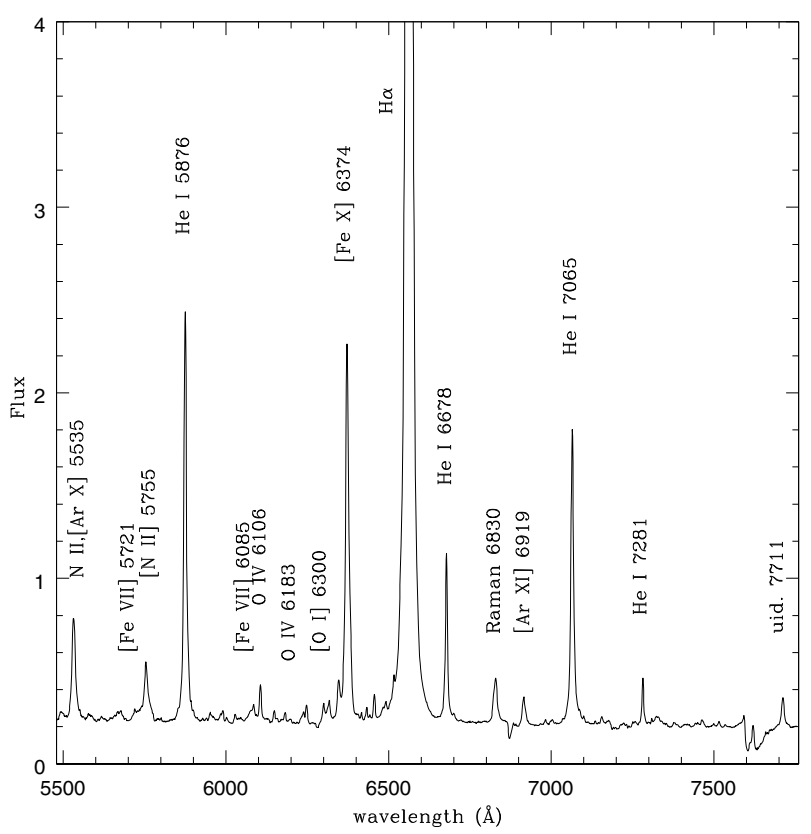

Fig. 18. Medium dispersion red spectrum on April 4. The unit of ordinate is $10^{-12} \mathrm{erg} \mathrm{cm}^{-2} \mathrm{~s}^{-1} \AA^{-1}$.

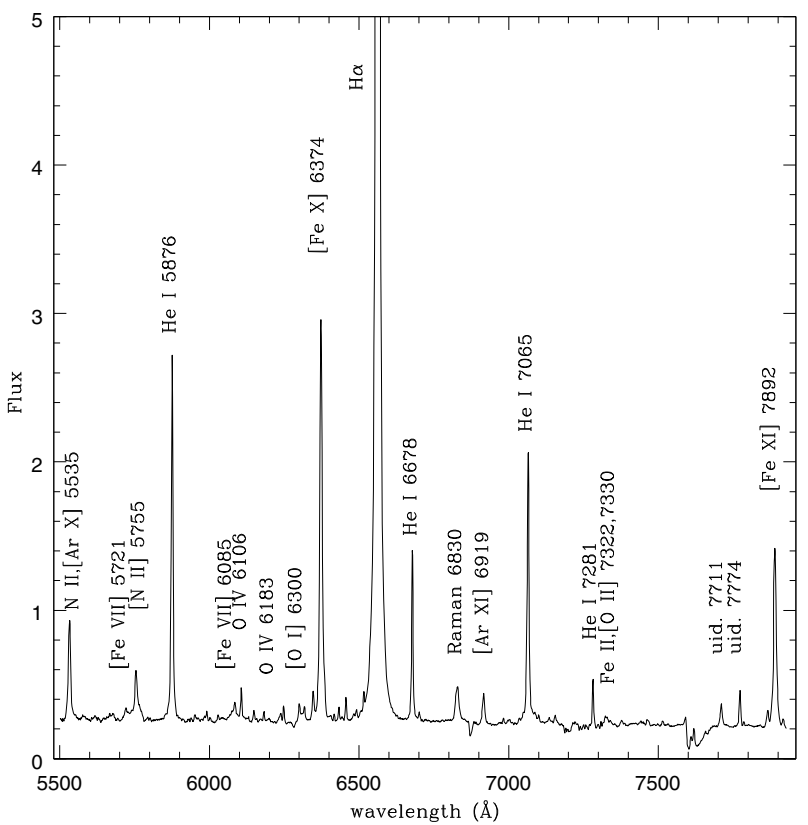

Fig. 19. Medium dispersion red spectrum on April 24. The unit of ordinate is $10^{-12} \mathrm{erg} \mathrm{cm}^{-2} \mathrm{~s}^{-1} \AA^{-1}$.

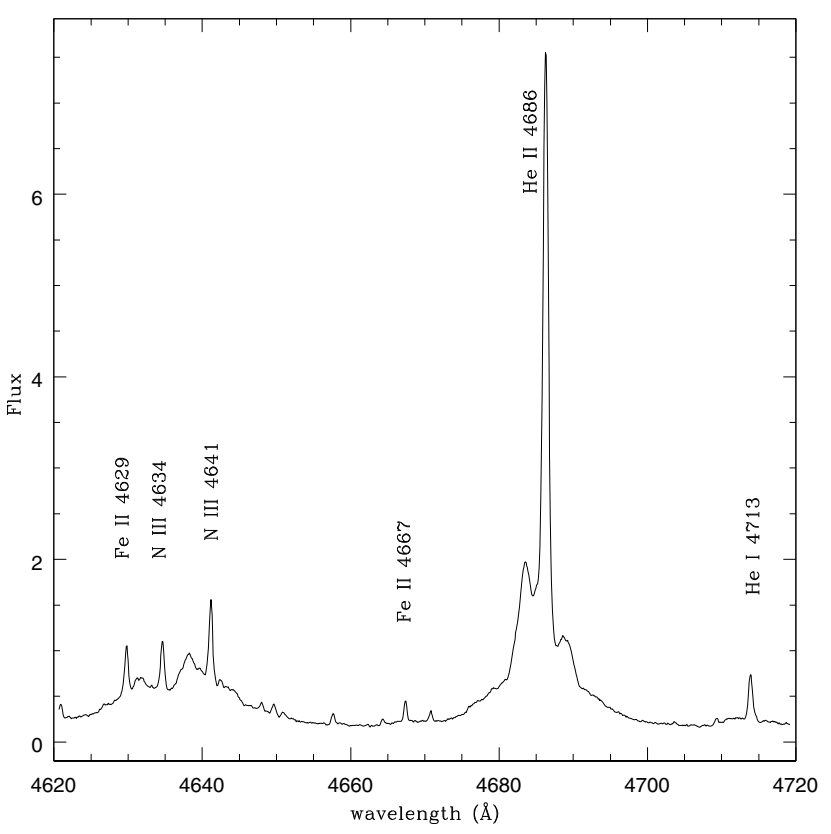

Fig. 20. High dispersion spectrum on April 8: 4620-4720A. The unit of ordinate is $10^{-12} \mathrm{erg} \mathrm{cm}^{-2} \mathrm{~s}^{-1} \AA^{-1}$. 
T. Iijima: Spectral evolution of RS Oph on the outburst in 2006, Online Material $p 7$

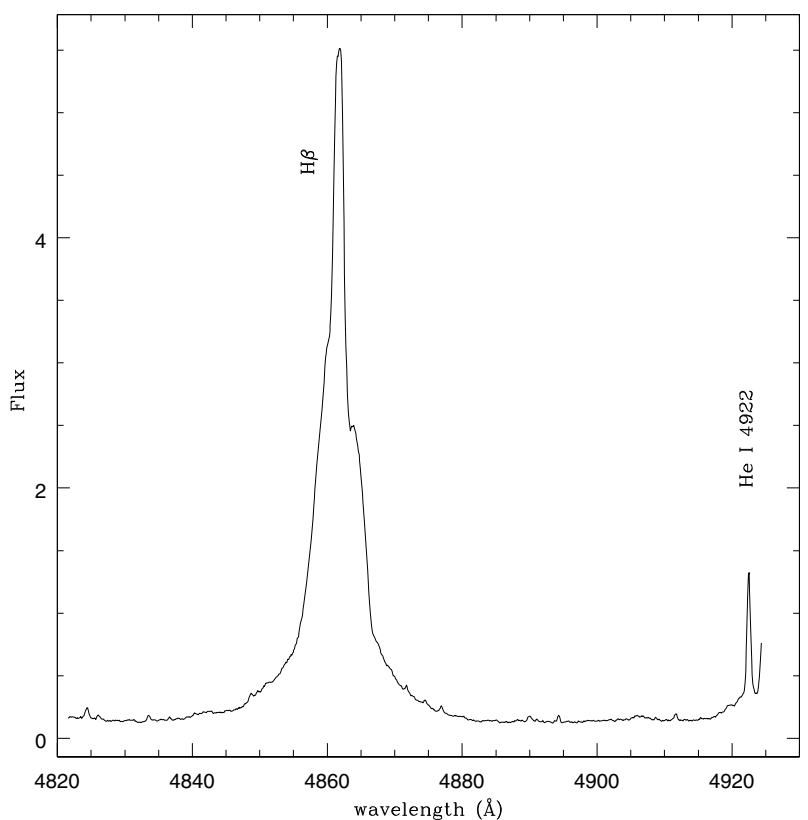

Fig. 21. High dispersion spectrum on April 8: profile of $\mathrm{H} \beta$. The unit of the ordinate is $10^{-12} \mathrm{erg} \mathrm{cm}^{-2} \mathrm{~s}^{-1} \AA^{-1}$.

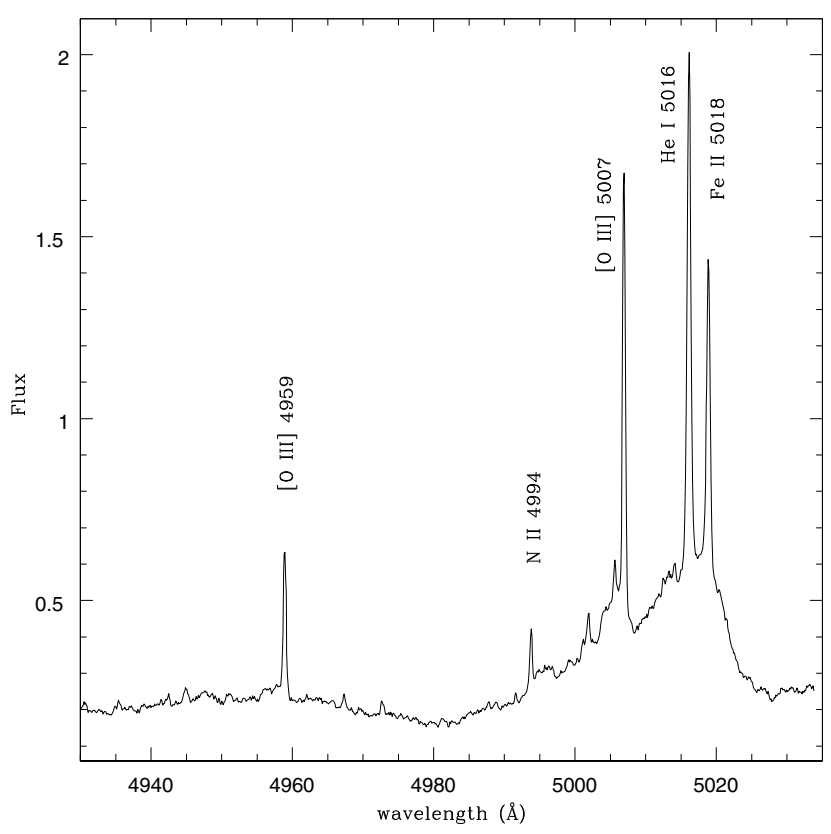

Fig. 22. High dispersion spectrum on April 8: 4930-5030A. The unit of ordinate is $10^{-12} \mathrm{erg} \mathrm{cm}^{-2} \mathrm{~s}^{-1} \AA^{-1}$.

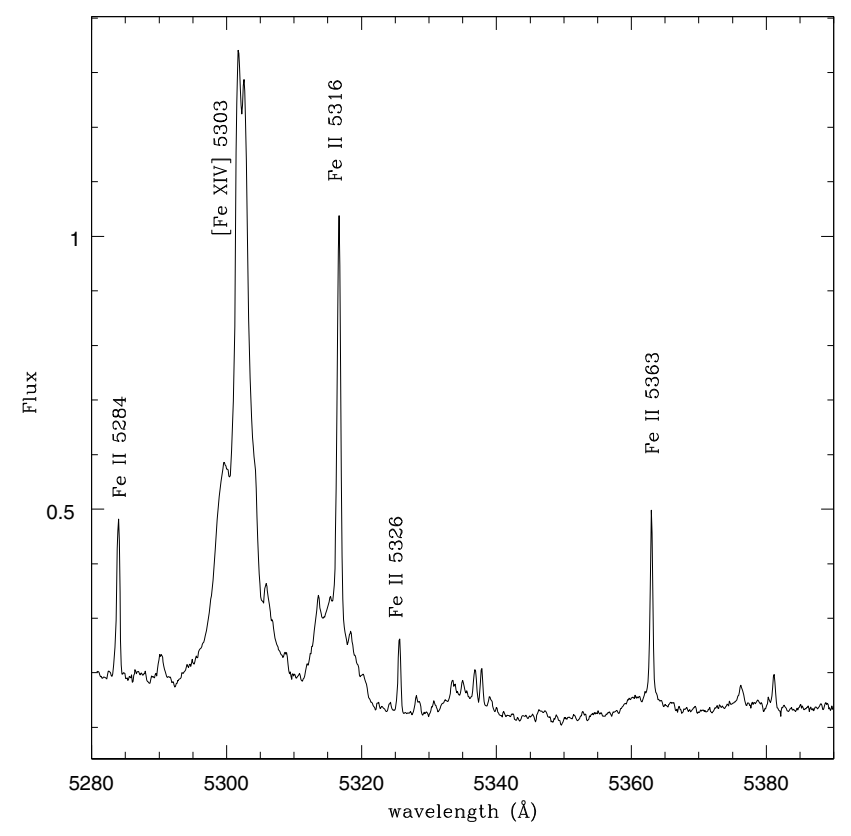

Fig. 23. High dispersion spectrum on April 8: 5280-5390Å. The unit of ordinate is $10^{-12} \mathrm{erg} \mathrm{cm}^{-2} \mathrm{~s}^{-1} \AA^{-1}$.

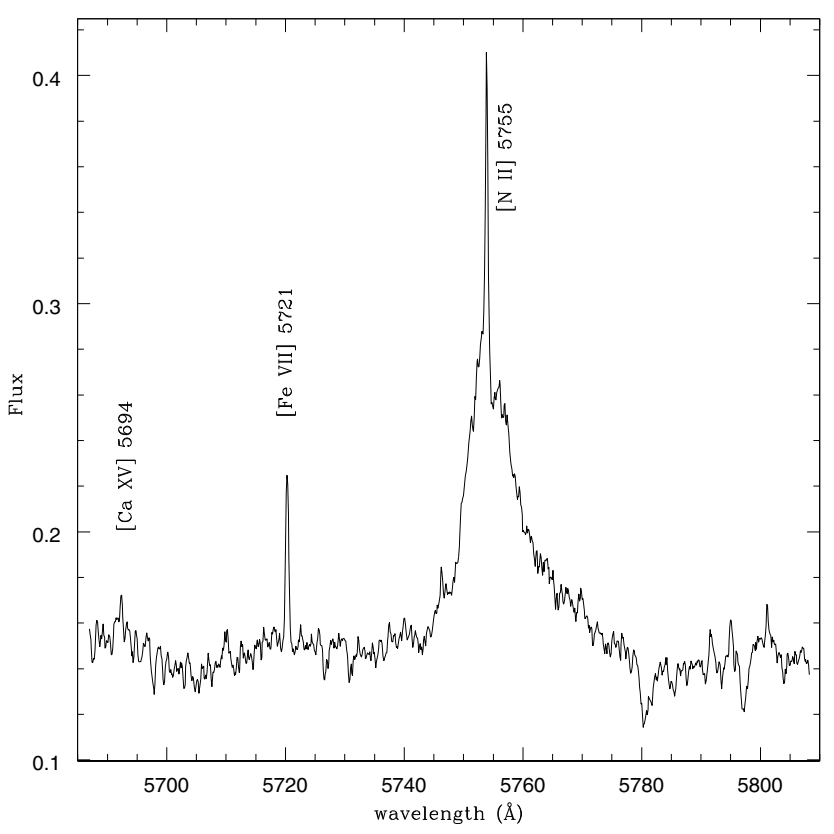

Fig. 24. High dispersion spectrum on April 8: 5690-5810Å. The unit of ordinate is $10^{-12} \mathrm{erg} \mathrm{cm}^{-2} \mathrm{~s}^{-1} \AA^{-1}$. 
T. Iijima: Spectral evolution of RS Oph on the outburst in 2006, Online Material $p 8$

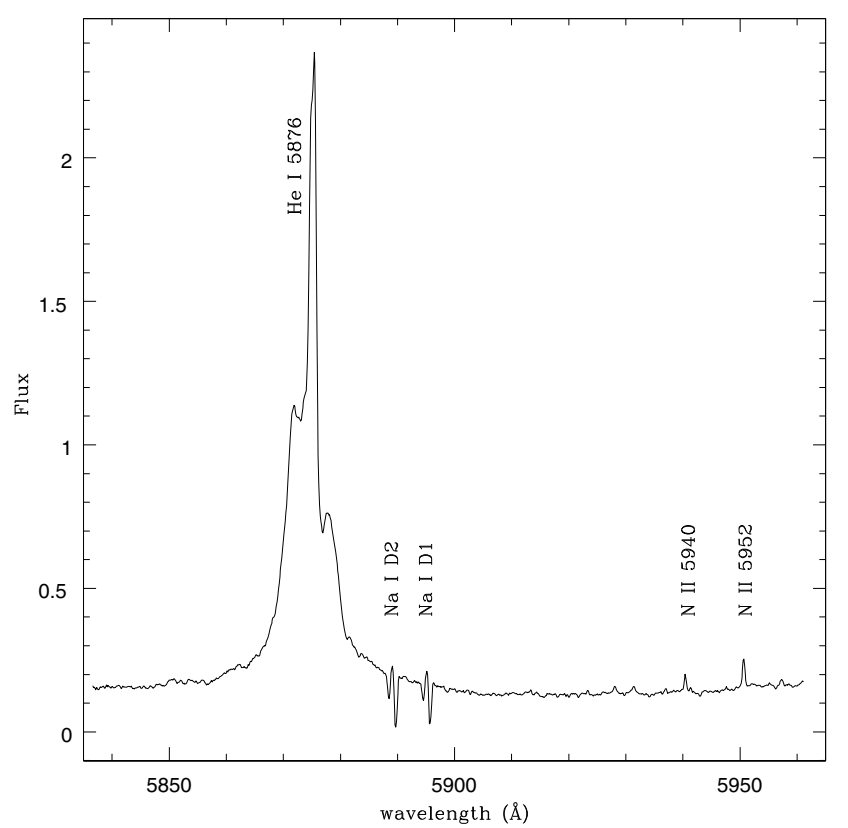

Fig. 25. High dispersion spectrum on April 8: 5840-5960A. The unit of ordinate is $10^{-12} \mathrm{erg} \mathrm{cm}^{-2} \mathrm{~s}^{-1} \AA^{-1}$.

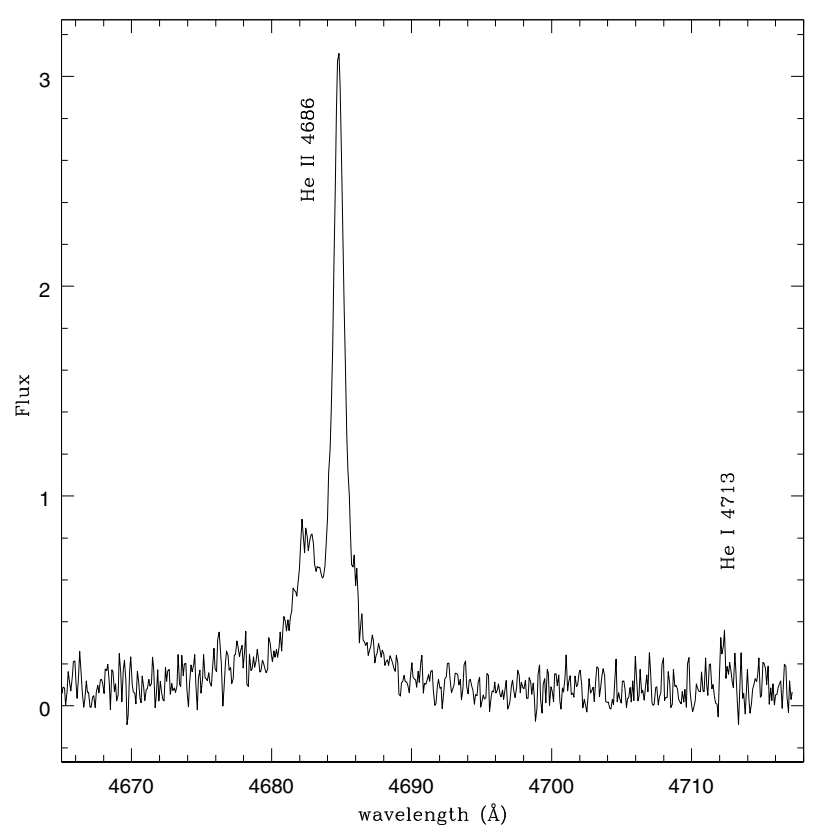

Fig. 26. High dispersion spectrum on May 17: profile of He II 4686. The unit of the ordinate is $10^{-12} \mathrm{erg} \mathrm{cm}^{-2} \mathrm{~s}^{-1} \AA^{-1}$.

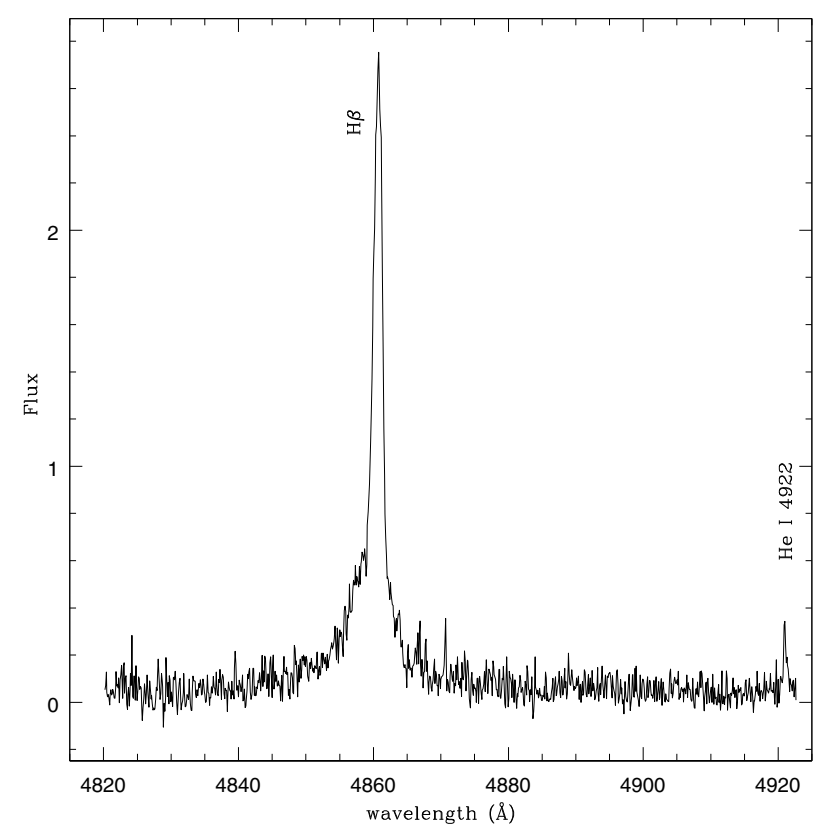

Fig. 27. High dispersion spectrum on May 17: profile of $\mathrm{H} \beta$. The unit of ordinate is $10^{-12} \mathrm{erg} \mathrm{cm}^{-2} \mathrm{~s}^{-1} \AA^{-1}$.

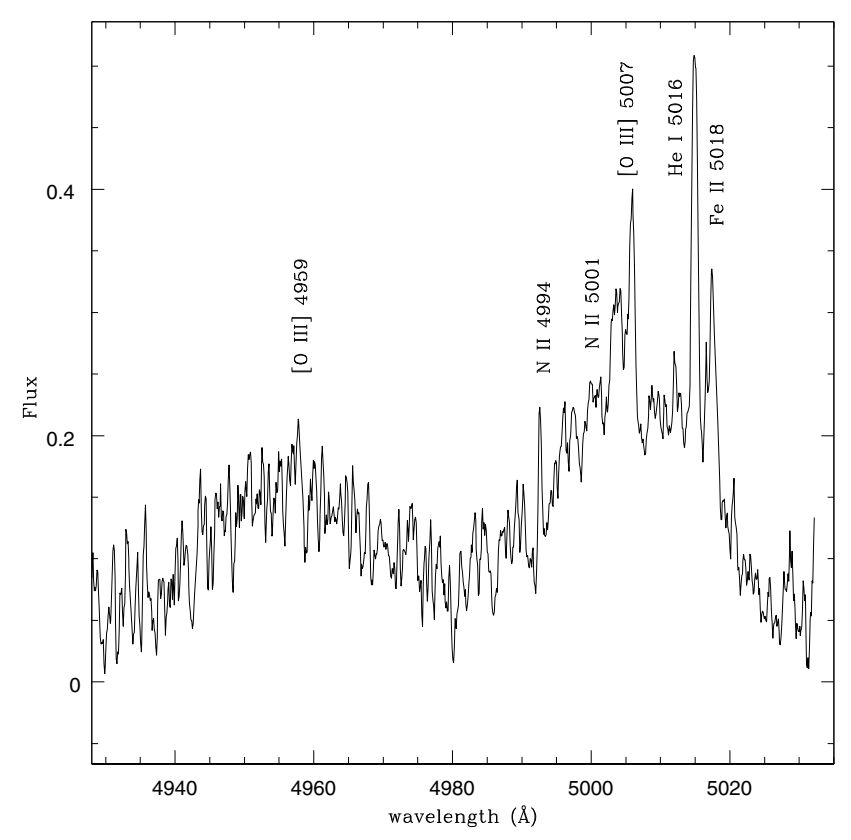

Fig. 28. High dispersion spectrum on May 17: 4930-5030 ̊. The unit of ordinate is $10^{-12} \mathrm{erg} \mathrm{cm}^{-2} \mathrm{~s}^{-1} \AA^{-1}$. 
T. Iijima: Spectral evolution of RS Oph on the outburst in 2006, Online Material $p 9$

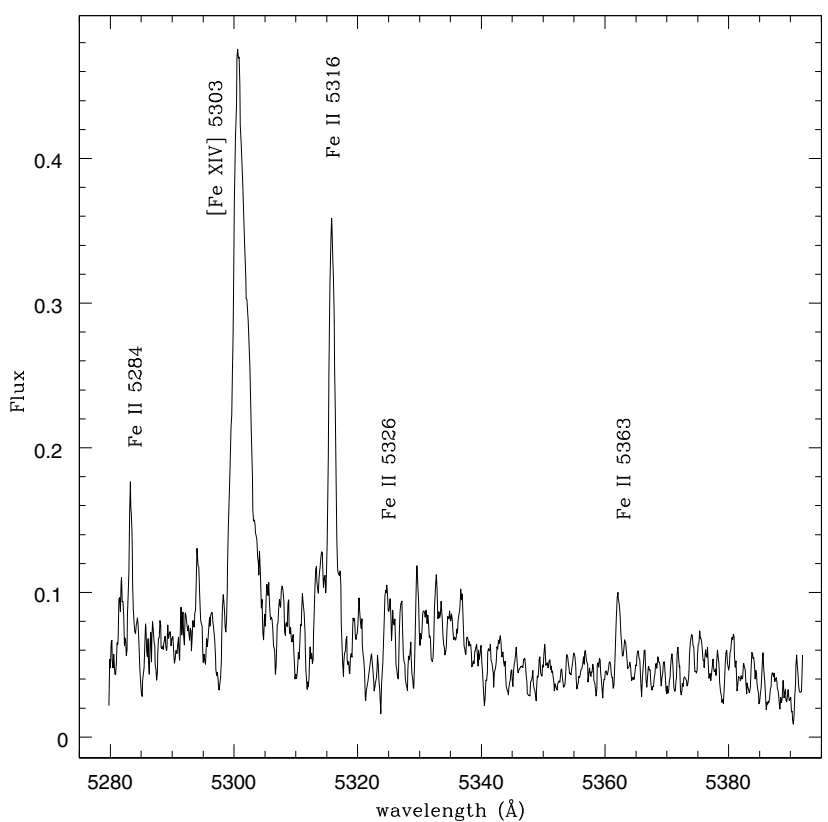

Fig. 29. High dispersion spectrum on May 17: 5280-5390A. The unit of ordinate is $10^{-12} \mathrm{erg} \mathrm{cm}^{-2} \mathrm{~s}^{-1} \AA^{-1}$.

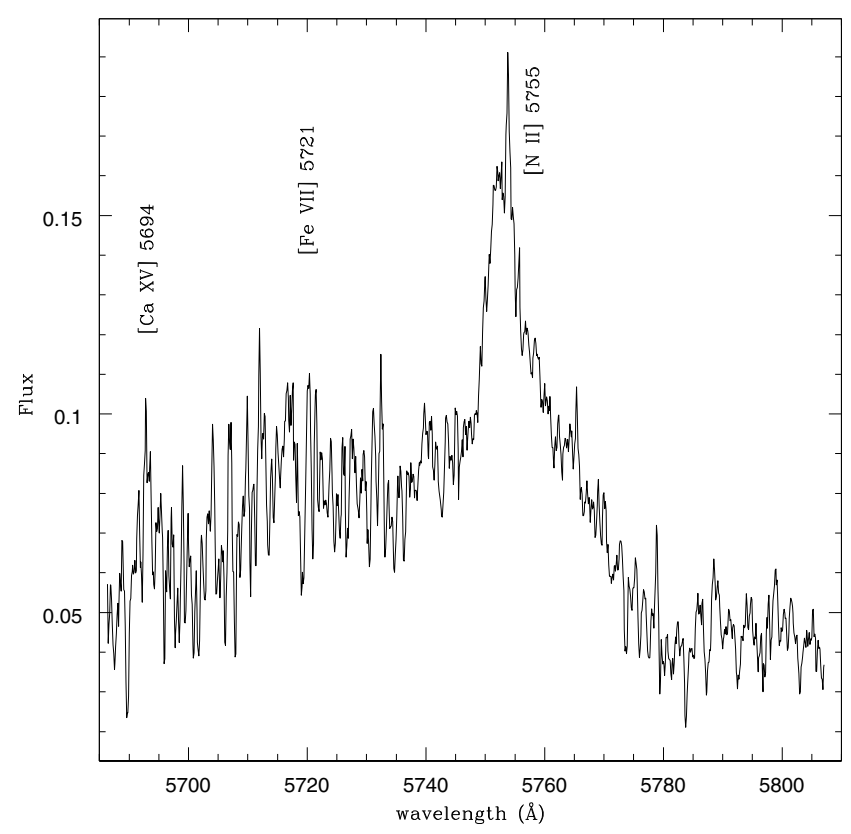

Fig. 30. High dispersion spectrum on May 17: 5690-5810A. The unit of ordinate is $10^{-12} \mathrm{erg} \mathrm{cm}^{-2} \mathrm{~s}^{-1} \AA^{-1}$.

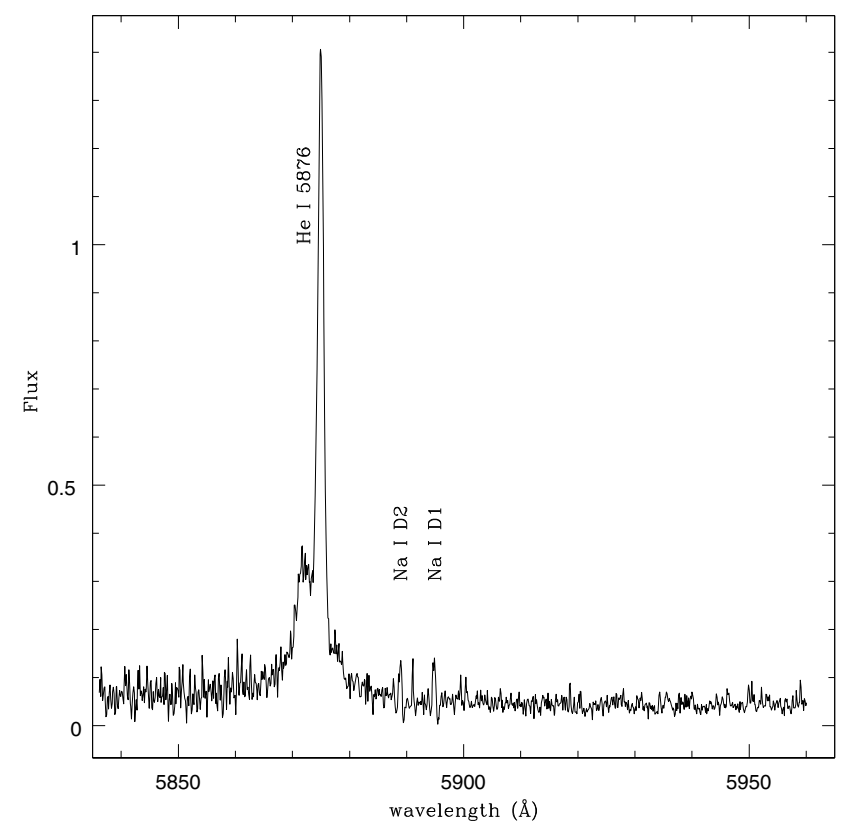

Fig. 31. High dispersion spectrum on May 17: 5840-5955 $\mathrm{A}$. The unit of ordinate is $10^{-12} \mathrm{erg} \mathrm{cm}^{-2} \mathrm{~s}^{-1} \AA^{-1}$.

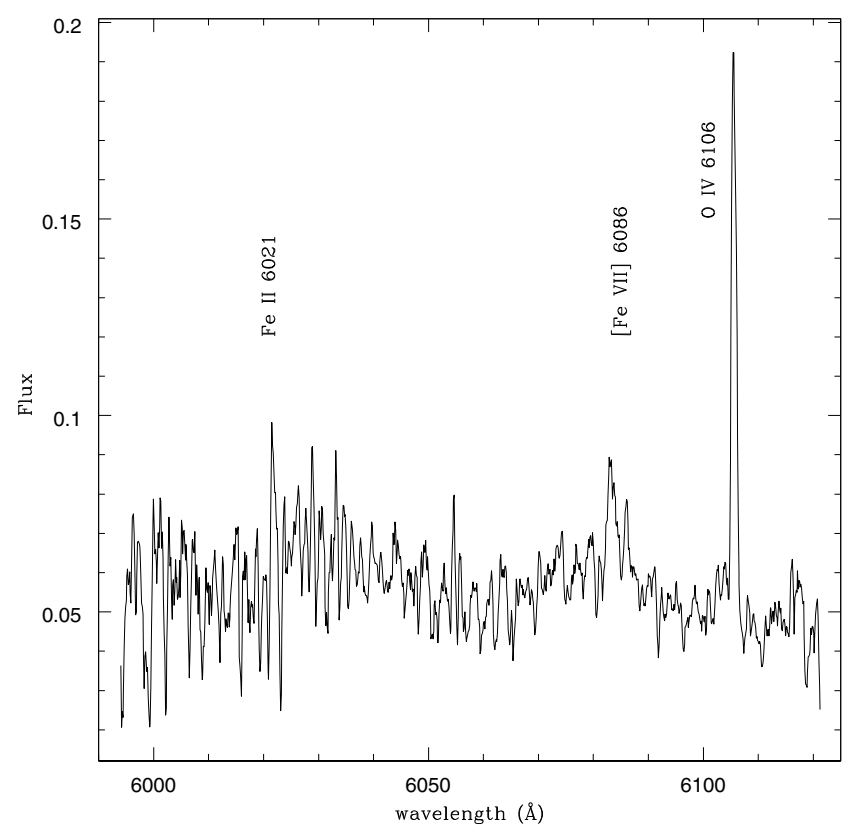

Fig. 32. High dispersion spectrum on May 17: 6000-6120 . The unit of ordinate is $10^{-12} \mathrm{erg} \mathrm{cm}^{-2} \mathrm{~s}^{-1} \AA^{-1}$. 
T. Iijima: Spectral evolution of RS Oph on the outburst in 2006, Online Material $p 10$

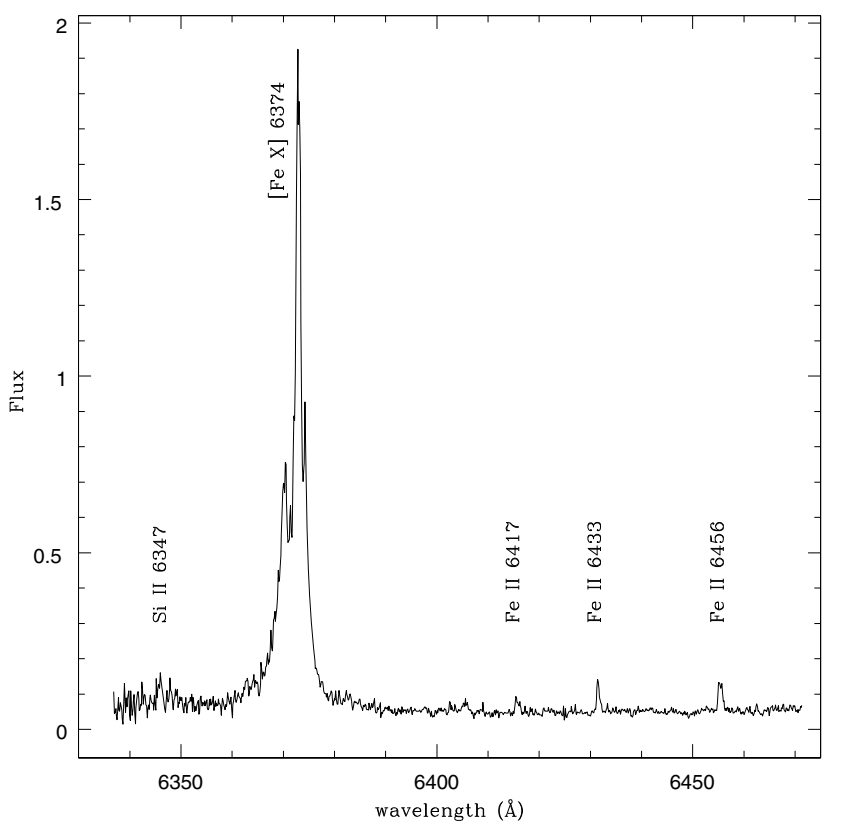

Fig. 33. High dispersion spectrum on May 17: 6340-6470A. The unit of ordinate is $10^{-12} \mathrm{erg} \mathrm{cm}^{-2} \mathrm{~s}^{-1} \AA^{-1}$.

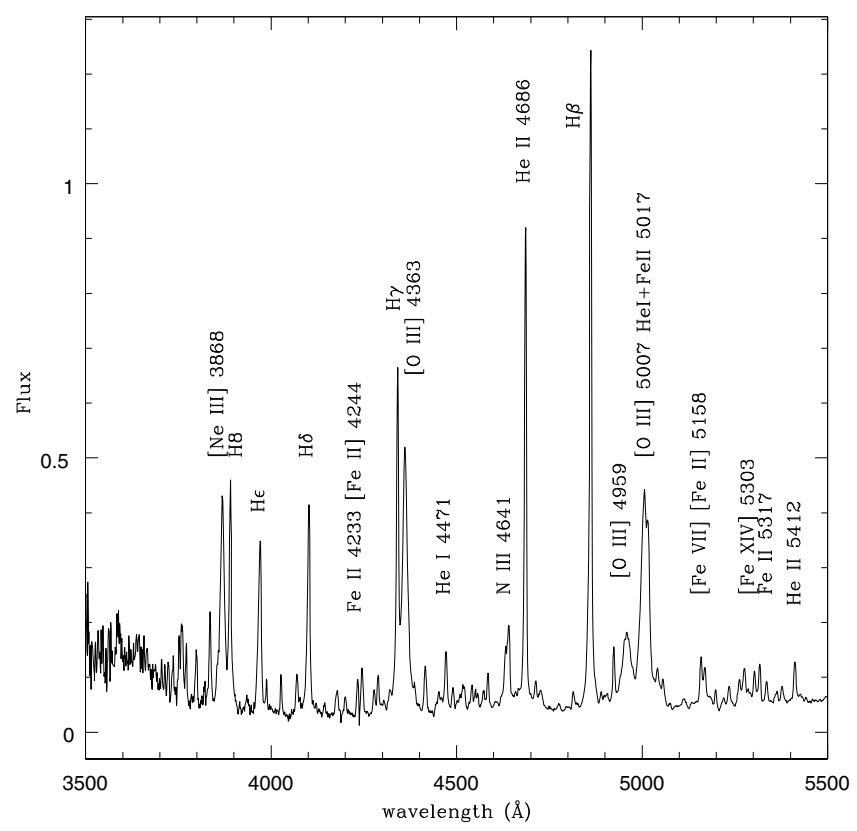

Fig. 34. Medium dispersion blue spectrum on May 26. The unit of ordinate is $10^{-12} \mathrm{erg} \mathrm{cm}^{-2} \mathrm{~s}^{-1} \AA^{-1}$.

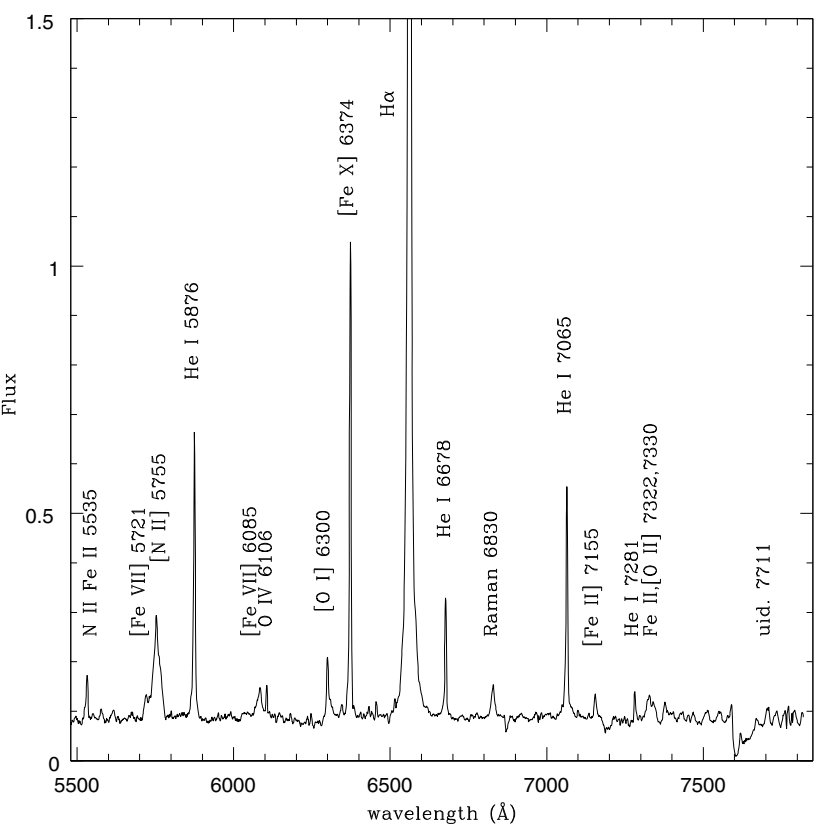

Fig. 35. Medium dispersion red spectrum on May 25. The unit of ordinate is $10^{-12} \mathrm{erg} \mathrm{cm}^{-2} \mathrm{~s}^{-1} \AA^{-1}$.

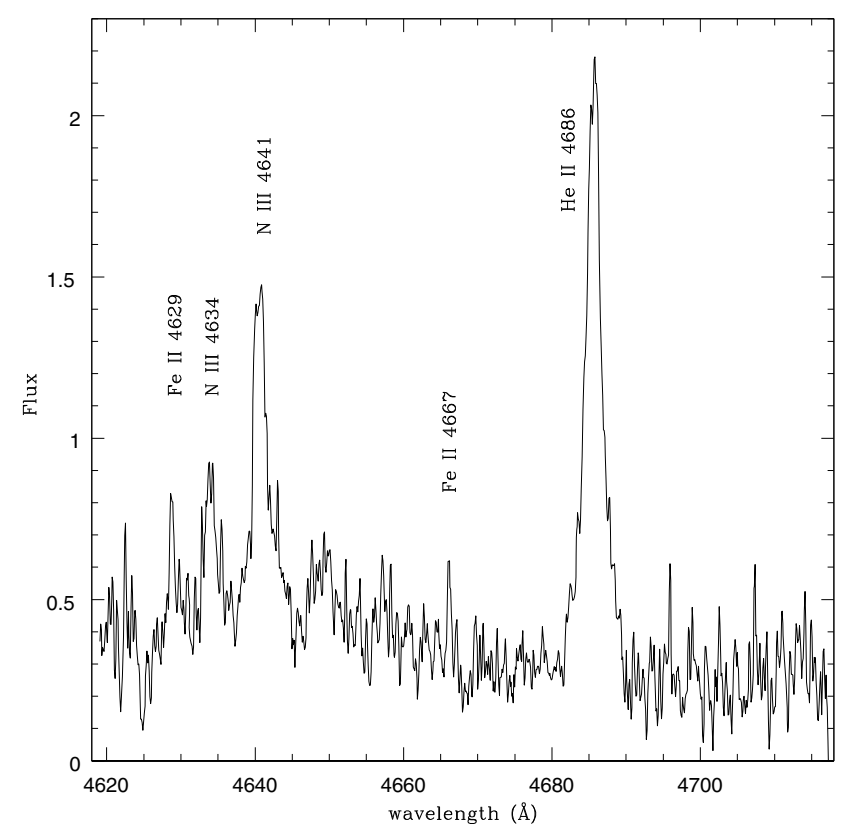

Fig. 36. High dispersion spectrum on August 31: 4620-4720Å. The unit of the ordinate is $10^{-12} \mathrm{erg} \mathrm{cm}^{-2} \mathrm{~s}^{-1} \AA^{-1}$. 
T. Iijima: Spectral evolution of RS Oph on the outburst in 2006, Online Material p 11

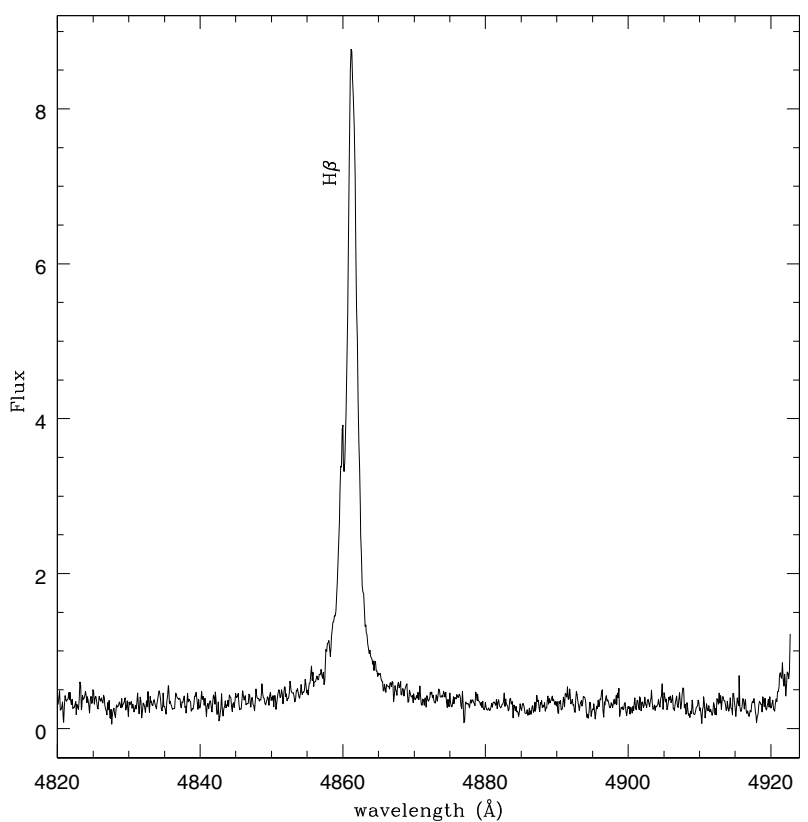

Fig. 37. High dispersion spectrum on August 31: profile of $\mathrm{H} \beta$. The unit of the ordinate is $10^{-12} \mathrm{erg} \mathrm{cm}^{-2} \mathrm{~s}^{-1} \AA^{-1}$.

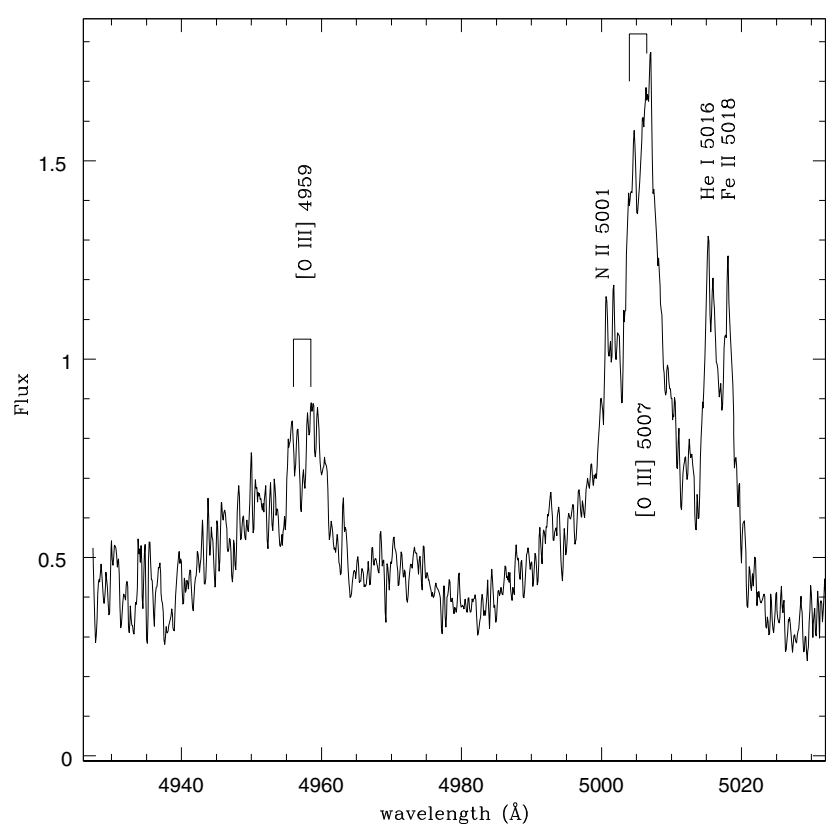

Fig. 38. High dispersion spectrum on August 31: 4930-5030 ̊. The unit of the ordinate is $10^{-12} \mathrm{erg} \mathrm{cm}^{-2} \mathrm{~s}^{-1} \AA^{-1}$.

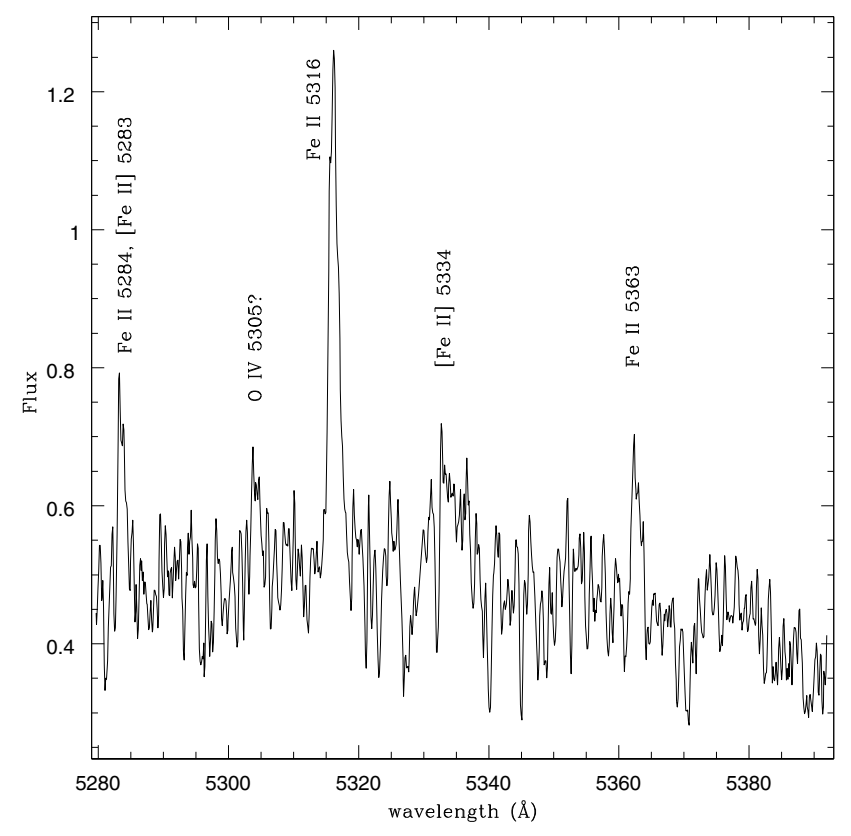

Fig. 39. High dispersion spectrum on August 31: 5280-5390. The unit of the ordinate is $10^{-12} \mathrm{erg} \mathrm{cm}^{-2} \mathrm{~s}^{-1} \AA^{-1}$.

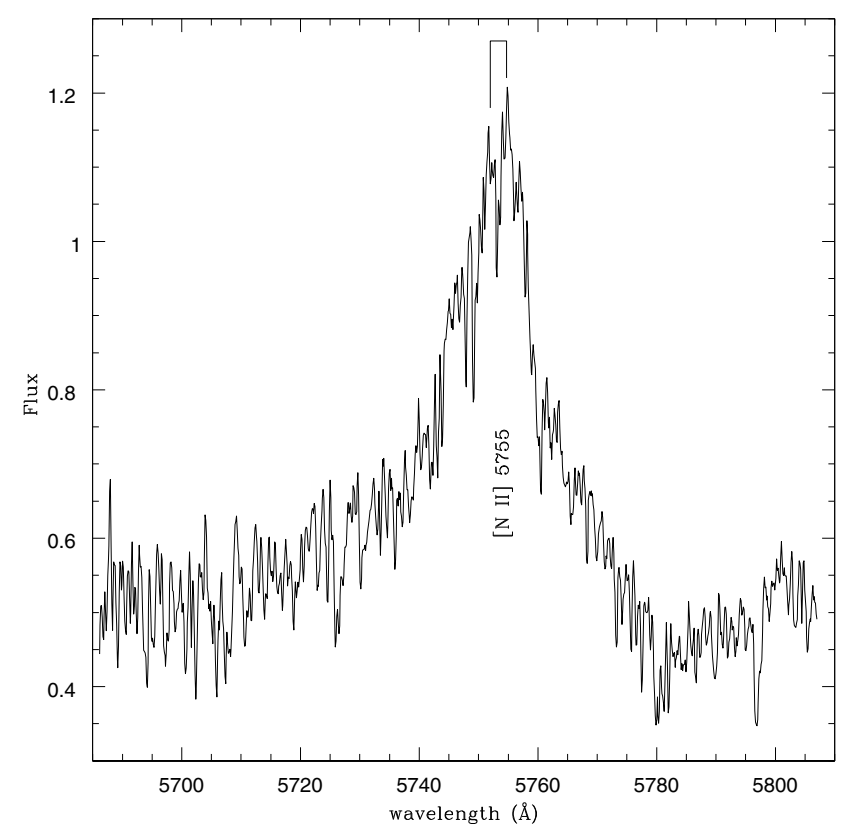

Fig. 40. High dispersion spectrum on August 31: 5690-5805 $\AA$. The unit of the ordinate is $10^{-12} \mathrm{erg} \mathrm{cm}^{-2} \mathrm{~s}^{-1} \AA^{-1}$. 
T. Iijima: Spectral evolution of RS Oph on the outburst in 2006, Online Material p 12

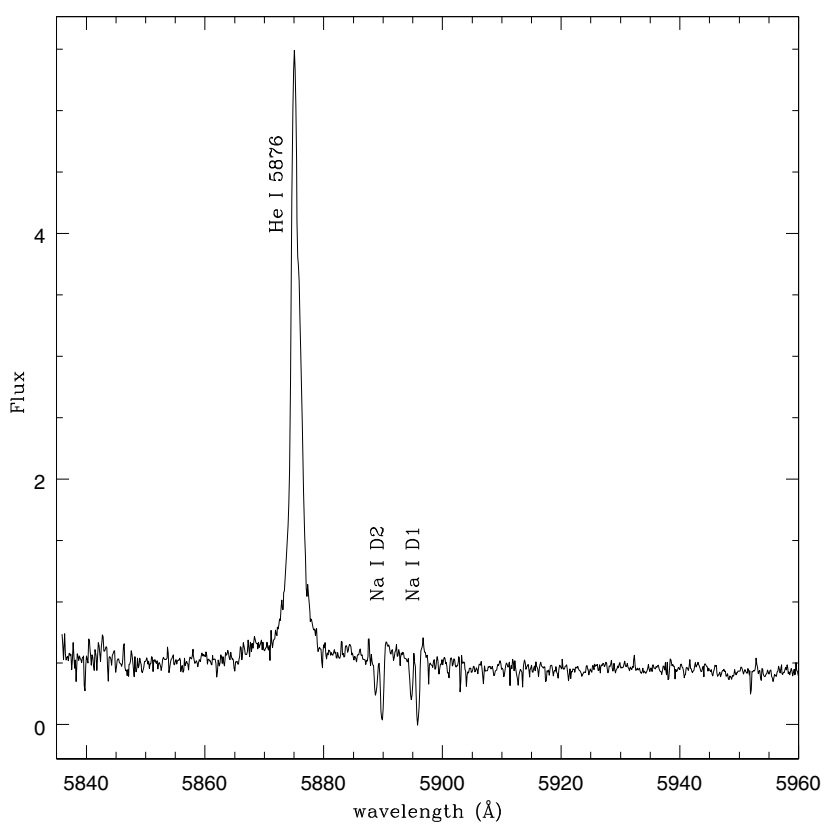

Fig. 41. High dispersion spectrum on August 31: 5840-5960. The unit of the ordinate is $10^{-12} \mathrm{erg} \mathrm{cm}^{-2} \mathrm{~s}^{-1} \AA^{-1}$.

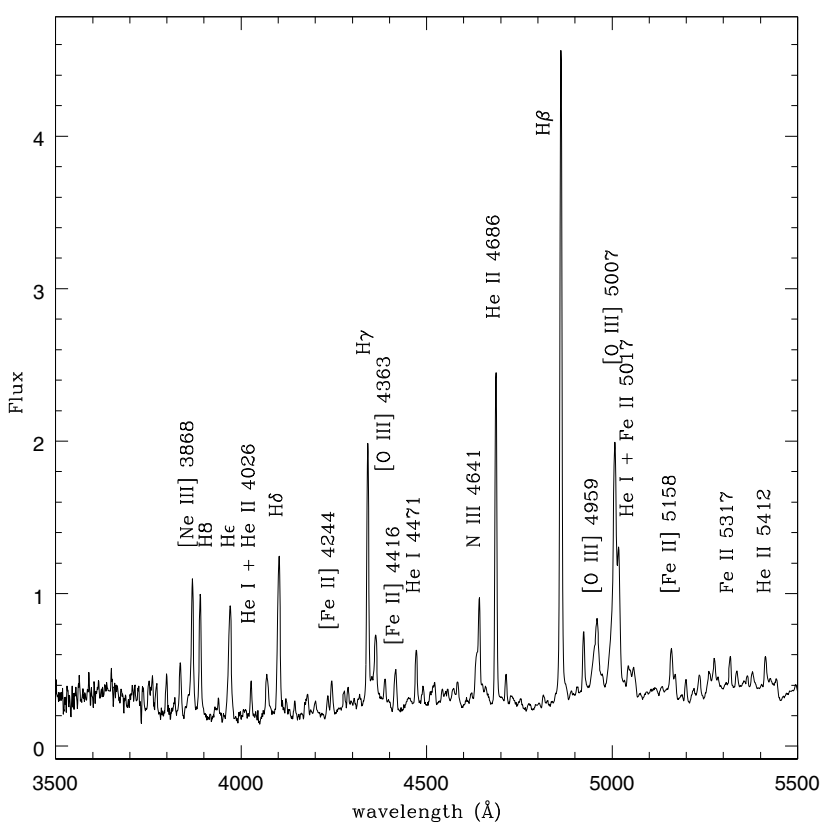

Fig. 42. Medium dispersion blue spectrum on September 9. The unit of ordinate is $10^{-12} \mathrm{erg} \mathrm{cm}^{-2} \mathrm{~s}^{-1} \AA^{-1}$.

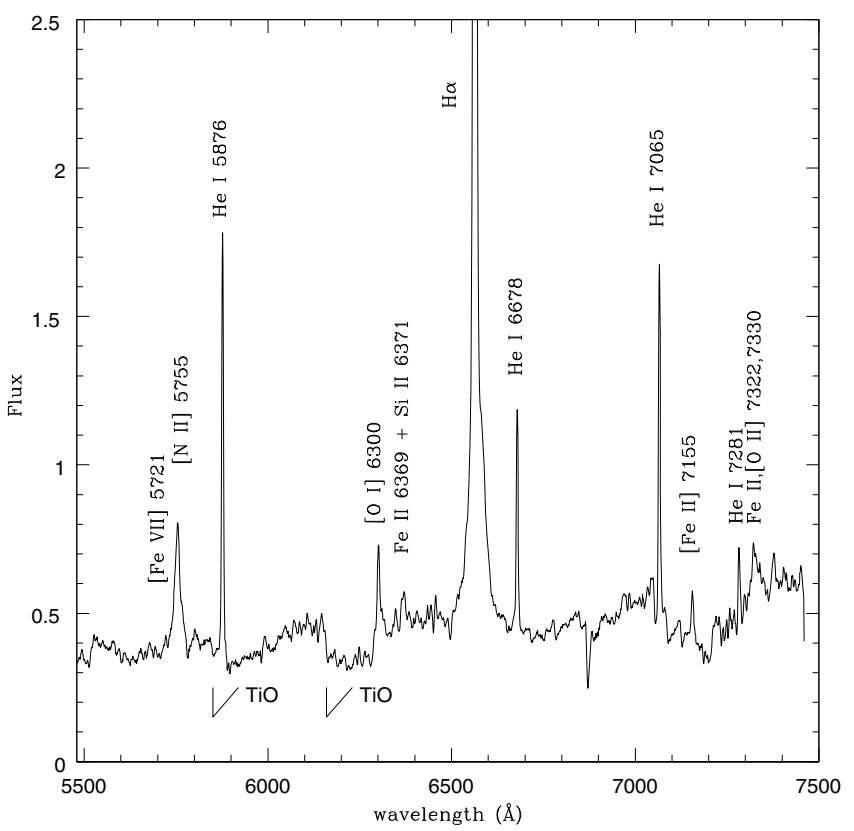

Fig. 43. Medium dispersion red spectrum on September 12. The unit of ordinate is $10^{-12} \mathrm{erg} \mathrm{cm}^{-2} \mathrm{~s}^{-1} \AA^{-1}$.

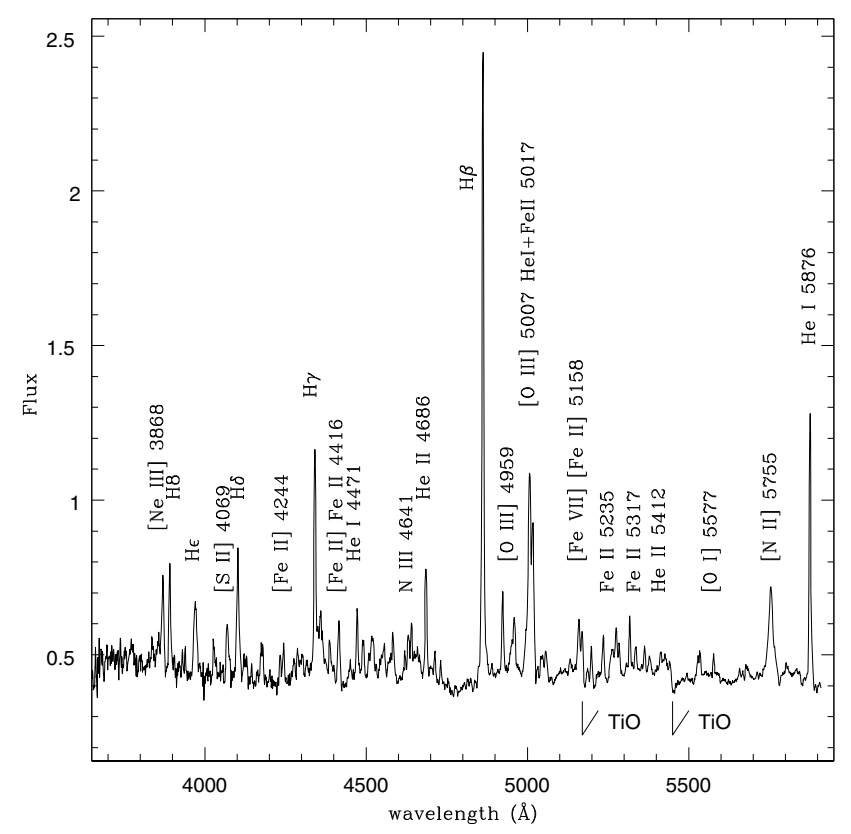

Fig. 44. Medium dispersion blue spectrum on October 29. The unit of ordinate is $10^{-13} \mathrm{erg} \mathrm{cm}^{-2} \mathrm{~s}^{-1} \AA^{-1}$. 